Revue d'histoire de l'Amérique française

REVUE D.HISTOIRE DE L'AMÉRIQUE FRANÇAISE

\title{
Les migrants américains et franco-américains au Québec, 1792-1940 : un état de la question
}

\section{Paul-André Linteau}

Volume 53, numéro 4, printemps 2000

Histoire des Premières Nations : nouvelles lectures et nouveaux problèmes

URI : https://id.erudit.org/iderudit/005536ar

DOI : https://doi.org/10.7202/005536ar

Aller au sommaire du numéro

Éditeur(s)

Institut d'histoire de l'Amérique française

ISSN

0035-2357 (imprimé)

1492-1383 (numérique)

Découvrir la revue

Citer cet article

Linteau, P.-A. (2000). Les migrants américains et franco-américains au Québec, 1792-1940 : un état de la question. Revue d'histoire de l'Amérique française, 53(4), 561-602. https://doi.org/10.7202/005536ar
Résumé de l'article

Les migrations des États-Unis vers le Québec sont au cœur de cet article. L'examen de l'historiographie permet d'abord de proposer une périodisation de l'immigration des Américains, chaque étape étant caractérisée par un type d'immigrant: le colon (1792-1820), l'entrepreneur (1820-1880), le gestionnaire et l'expert (1880-1940). L'importance du retour au Québec de centaines de milliers de Franco-Américains est ensuite mise en lumière. L'étude des biographies de membres des élites révèle la diversité de leurs trajectoires nord-américaines. Pour les autres composantes de la population, un bilan des recherches récentes est proposé. L'auteur conclut cet état de la question en soulignant l'importance de ce double mouvement migratoire pour l'interprétation de l'histoire du Québec et suggère des pistes de recherches futures. 


\section{L es migrants américains et franco- américains au Q uébec, 1792-1940:}

un état de la question ${ }^{1}$

PAUL-ANDRÉ LINTEAU

Département d'histoire

Université du Québec à Montréal

r ésu mé - Les migrations des États-Unis vers le Q uébec sont au cœur de cet aticle. L'examen de l'historiographie permet d'abord de proposer une périodisation de l'immigration des Américains, chaque étape étant caractérisée par un type dimmigrant: le colon (1792-1820), l'entrepreneur (1820-1880), le gestionnaire et l'expert (1880-1940). L'importance du retour au Q uébec de centaines de milliers de Franco-Américains est ensuite mise en lumière. L'étude des biographies de membres des élites révèle la diversité de leurs trajectoires nord-américaines. Pour les autres composantes de la population, un bilan des recherches récentes est proposé. L'auteur conclut cet état de la question en soulignant l'importance de ce double mouvement migatoire pour l'interprétation de I'histoire du Q uébec et suggère des pistes de recherches futures.

abstract . This article deals with the migation flows from the United States to $Q$ uébec. First, a historiographical survey leads to a chronology of American immigration, each period being represented by a leading immigant's type: the pioneer (1792-1820), the entrepreneur (1820-1880), the manager and expert (1880-1940). Second, the importance of the Franco-American return migation is highlighted. The diversity of the elites' $\mathrm{N}$ orth-American migrations is revealed through biographical research. For the other social groups, a survey of the recent literature is presented. The author concludes by stressing the significance of this double migratory movement for the history of Q uébec and by offering ideas for further research.

1. L'auteur remercie les collègues qui lui ont fait d'utiles commentaires et suggestions, notamment Gérard Bouchard, Madeleine Greffard, Fernand Harvey, José Igartua, Serge Jaumain, J. I. Little, M artin Pâquet, Bruno Ramirez, Jean-Claude Robert, Yves Roby et Fernande Roy ainsi que les évaluateurs de la Revue. 


\section{$\Lambda$ u cours des $\mathrm{xix}^{\mathrm{e}}$ et $\mathrm{xx}^{\mathrm{e}}$ siècles, le voisinage des États-Unis a touché le Québec dans une multitude de domaines: géographie partagée,} mouvements migratoires, communications, échanges commerciaux, investissements, transferts technologiques, relations syndicales, tourisme, éducation, création et diffusion artistiques et culturelles, mouvements d'idées, etc. ${ }^{2}$. Pourtant, l'étude des relations entre ces deux territoires et leurs populations n'occupe pas une très grande place dans I'historiographie. Certes, elle a suscité sporadiquement l'intérêt d'historiens et d'autres spécialistes et mené à diverses publications, dont une première synthèse encore très partielle, parue en $1991^{3}$. On est cependant loin d'avoir épuisé le sujet.

Le corpus historiographique le plus imposant conceme l'émigration des Canadiens français, surtout en Nouvelle-Angleterre. Si quelques travaux ont cherché à mesurer l'ampleur du phénomène ou encore à examiner les conditions qui expliquent les départs en masse, la majorité porte sur la Franco-Américanie elle-même: sa socio-économie, son cadre institutionnel et les débats politico-religieux qui ont marqué son histoire 4 . On ne s'est guère intéressé à en étudier l'effet de rétroaction, c'est-à-dire l'impact de ce mouvement migratoire sur la société québécoise elle-même. Sur ce plan, seule l'étude du discous des élites a retenu l'attention.

D'autres recherches sur la perception et l'influence des États-Unis dans la société québécoise ont surtout porté sur le champ culturel - en

2. Cette question a fait l'objet de premières formulations dans des textes parus à l'étranger et peu connus au Québec: Paul-André Linteau, «Le relazioni tra il Québec e gli Stati Uniti nel xıxe xx secolo : storiografia e prospettive di ricerca», V. Gennaro Lerda, dir., Canadiana, Canada eStati Uniti (Venise, Marsilio Editori, 1984), 87-105; id., «La identidad francófona de Quebec en un contexto norteamericano y multicultural : perspectivas históricas», Teresa Gutierrez H. et M onica Verea C., dir., Canadá en transición (M exico, Universidad Nacional Autonoma de M exico, 1994), 603-626; id., «Le Québec et les grands ensembles linguistiques et culturels: les rapports avec la France et les États-Unis depuis le milieu du xix ${ }^{e}$ siècle», Zeitschrift für Kanada-Studien, 17. Jahrgang/ Nr. 2, Band 32 (1997): 19-35.

3. Robert Chodos et Eric Hamovitch, Québec and the American Dream (Toronto, Between the Lines, 1991), $251 \mathrm{p}$.

4. La production historiographique sur les Franco-Américains est considérable. Limitonsnous ici à citer les deux principales synthèses: Yves Roby, Les Franco-Américains de la NouvelleAngleterre, 1776-1930 (Sillery, Septentrion, 1990), 434 p. ; François Weil, Les Franco-Américains (Paris, Belin, 1989), 251 p. ; pour l'expansion canadienne-française hors de la NouvelleAngleterre, voir aussi D. Aidan McQuillan, «Les communautés canadiennes-françaises du Midwest américain au dix-neuvième siècle», Dean R. Louder et Eric Waddell, dir., Du continent perdu à l'archipel retrouvé. Le Québec et l'Amérique française (Québec, Les Presses de I'U niversité Laval, 1983), 97-115 et Yves Frenette, Brève histoi re des Canadiens français (M ontréal, Boréal, 1998), $210 \mathrm{p}$. 
particulier les arts et la littérature - et sur les mouvements d'idées. Deux colloques importants ont abordé ces questions et leurs travaux ont été publiés ${ }^{5}$. Des monographies ont été consacrées à des manifestations de l'américanisation de la culture populaire ${ }^{6}$ ou à l'attitude des élites envers la société américaine. Par ailleurs, les investissements américains au Québec ont suscité quelque intérêt, notamment gâce à Albert Faucher ${ }^{8}$, mais les études spécifiques sont encore peu nombreuses: elles ont surtout porté sur l'industrie papetière ${ }^{9}$ ainsi que sur les réactions exprimées par des Québécois à l'endroit de ce phénomène ${ }^{0}$. Sans être le principal objet d'attention, la présence américaine au Québec est aussi évoquée dans un grand nombre de travaux portant sur l'histoire de villes ou de régions, de secteurs d'activité ou d'institutions spécifiques.

N ous proposons d'ajouter un autre élément à la compréhension des rapports américano-québécois en nous penchant sur la présence en sol québécois des Américains eux-mêmes En effet, tout au long des xix ${ }^{\mathrm{e}}$ et $\mathrm{xx}^{\mathrm{e}}$ siècles, le Québec a régulièrement accueilli des migrants ${ }^{11}$ permanents ou temporaires en provenance des États-Unis Qui sont-ils, d'où viennent-ils, où vont-ils? Dans ce mouvement migratoire, quelle est la part des Américains proprement dits et celle des Franco-Américains? Si la présence américaine au Canada a déjà été étudiée, notamment par David D. Harvey ${ }^{12}$, la situation du Québec n'y est pas traitée de façon spécifique.

5. Claude Savary, dir., Les rapports culturels entre le Québec et les États-Unis (Q uébec, Institut québécois de recherche sur la culture, 1984), 353 p. ; Gérard Bouchard et Yvan Lamonde, Qué bécois et Américains. La culture québécoise aux xix et $x x^{e}$ siècles (Saint-Laurent, Fides, 1995), $418 p$.

6. Par exemple, Chantal Hébert, Le burlesque au Québec, un divertissement populaire (M ontréal, H urtubise HMH, 1981), 302 p. ; Donald Cuccioletta, The Américanité of Quebec Urban Popular Culture As Seen Through Burlesque Theater in Montreal (1919-1939), thèse de doctorat (histoire), Université du Québec à Montréal, 1997, 337 p.

7. Guildo Rousseau, L'image des États-Unis dans la littérature québécoise (1775-1930) (Sherbrooke, Naaman, 1981), 356 p. ; Yvan Lamonde, Ni avec eux ni sans eux. Le Québec et les États-U nis (Saint-Lambert, Nuit blanche, 1996), $121 \mathrm{p}$.

8. Albert Faucher, «Le caractère continental de l'industrialisation au Québec», Histoi re économique et unité canadienne (M ontréal, Fides, 1970), 161-178.

9. Jorge N iosi, «La Laurentide (1887-1928): pionnière du papier journal au Canada», RHAF, 29,3 (décembre 1975): 375-415; Gilles Piédalue, «Les groupes financiers et la guerre du papier au Canada 1920-1940», RHAF, 30,2 (septembre 1976) : 223-258.

10. Yves Roby, Les Québécois et les investissements américains (1918-1929) (Québec, Les Presses de l'Université Laval, 1976), $250 \mathrm{p}$.

11. Le terme «migrants» est employé ici dans son sens le plus général : il englobe aussi bien les immigrants (et les émigrants) que les participants aux autres mouvements de population, de nature saisonnière ou temporaire.

12. David D. Harvey, Americans in Canada: Migration and Settlement since 1840 (Queenston [Ontario], Edwin Mellen Press, 1991), 442 p. 
Notre objectif est de dresser un état des connaissances et de dégager les principales caractéristiques de ce phénomène, en nous concentrant sur la période qui va de 1792 à 1940. Or, souvent limitée à l'étude d'individus ou de régions, l'historiographie pertinente reste éclatée, car très peu d'auteurs ont abordé directement l'examen de la présence des migrants américains et franco-américains en sol québécois Notre démarche s'appuie sur une relecture de cette production historique afin d'en dégager une signification cohérente du point de vue de notre objet d'étude. Dans le cas des migrants franco-américains, nous irons un peu plus loin en livrant les résultats de nos propres recherches dans les répertoires biographiques de la fin du $\mathrm{xIx}^{\mathrm{e}}$ et du début $\mathrm{du} \mathrm{xx}^{\mathrm{e}}$ siècle. Auparavant, il importe d'essayer de mesurer les effectifs en cause.

\section{él éments de mesure}

Combien d'Américains sont venus s'établir au Québec entre le début du $\mathrm{xIx}^{\mathrm{e}}$ siècle et le milieu du $\mathrm{xx}^{\mathrm{e}}$ siècle? Parmi eux, combien étaient d'origine française? Les réponses à ces questions ne sont pas faciles à obtenir, en raison de l'état des sources, mais aussi de la porosité légendaire qui a longtemps caractérisé la frontière canado-américaine.

Jusqu'au milieu des années 1920, les statistiques canadiennes sur l'immigration en provenance des États-Unis soulèvent de sérieux problèmes de fiabilité. Elles reposent souvent sur des estimés et, pour certaines années, sont grossièrement exagérées ${ }^{13}$. Elles ne distinguent généralement pas les véritables immigrants des Canadiens rentrant au pays et n'indiquent pas toujours la province de destination finale. Pendant une grande partie de la période examinée ici, elles ne peuvent donc pas être utilisées pour l'étude d'une province en particulier et il faut se tourner vers les recensements.

Les données des recensements relatives à l'origine ethnique ne sont d'aucun secours, car il n'y existe pas de catégorie «américaine». En effet, pendant plus d'un siècle, la question posée aux recensés concernait l'origine de leur premier ancêtre masculin arrivé en Amérique, de sorte que les personnes venues des États-Unis devaient indiquer l'origine de cet ancêtre, européen ou autre.

La seule donnée utilisable est celle du pays de naissance des personnes recensées, une question toujours posée depuis 1844. Elle permet de

13. M. C. Urquhart et K. A. H. Buckley, dir., Historical Statistics of Canada (Toronto, Macmillan, 1965), 9-11. 
t a bleau 1

Population du Québec et du Canada

née aux États-Unis, 1884-1971

\begin{tabular}{lccccc}
\hline Année & \multicolumn{2}{c}{ Québec } & \multicolumn{2}{c}{ Canada } & $\begin{array}{c}\text { Québec/Canada } \\
\text { n }\end{array}$ \\
\hline 1844 & 11946 & 1,7 & & & \\
\hline 1851 & 12482 & 1,4 & & & \\
1861 & 13648 & 1,2 & & & \\
1871 & 14714 & 1,2 & 64613 & 1,8 & 22,8 \\
1881 & 19415 & 1,4 & 77753 & 1,8 & 24,9 \\
1891 & 18524 & 1,2 & 80915 & 1,7 & 22,9 \\
1901 & 28405 & 1,7 & 127899 & 2,4 & 22,2 \\
1911 & 29843 & 1,5 & 303680 & 4,2 & 9,8 \\
1921 & 42124 & 1,8 & 374024 & 4,3 & 11,3 \\
1931 & 49406 & 1,7 & 344574 & 3,3 & 14,3 \\
1941 & 50229 & 1,5 & 312473 & 2,7 & 16,1 \\
1951 & 42286 & 1,0 & 282010 & 2,0 & 15,0 \\
1961 & 45933 & 0,9 & 283908 & 1,6 & 16,2 \\
1971 & 46480 & 0,8 & 309640 & 1,4 & 15,0 \\
\hline
\end{tabular}

Source : Recensements du Canada.

savoir combien de personnes nées aux États-Unis habitaient le Canada ou le Québec au moment du recensement (tableau 1). Sauf pour un léger déclin en 1891, le nombre des Américains résidant au Québec augmente d'un recensement à l'autre jusqu'en 1941. II n'est cependant pas considérable et sa proportion dans la population oscille entre 1,2\% et $1,8 \%$, puis baisse nettement à partir de 1951. En examinant les effectifs, on observe trois poussées de croissance, tout de même assez modestes: dans les huitième et dizième décennies du xıx ${ }^{\mathrm{e}}$ siècle et dans la seconde du siècle suivant. Pendant le dernier tiers du $\mathrm{xix}^{\mathrm{e}}$ siècle, le Québec abrite entre $22 \%$ et $25 \%$ des Américains établis au Canada, mais ce pourcentage chute radicalement au début du $\mathrm{xx}^{\mathrm{e}}$ siècle - résultat de l'arrivée d'un fort contingent d'immigrants américains dans l'Ouest - , avant de remonter par la suite, pour se stabiliser à 15-16\%.

Le recensement de 1941 est le premier à fournir des indications quant à la répartition spatiale des Américains établis au Québec Ils sont alors surreprésentés dans les villes, puisque $77,6 \%$ sont urbains (contre $61,2 \%$ 
pour l'ensemble de la population) et que, parmi ceux-ci, les trois cinquièmes habitent des centres de 30000 habitants ou plus ${ }^{4}$. De son côté, le recensement de 1921 livre une information plus restreinte, soit le pourcentage de personnes nées aux États-Unis dans la population d'une vingtaine de villes québécoises Ce chiffre atteint 5,5\% à Westmount, $5,0 \%$ à Grand-M ère, 4,9\% à O utremont, 4,6\% à Sherbrooke, mais 2,5\% à Montréal et seulement $0,9 \%$ à Québec ${ }^{15}$. II existe donc de grandes disparités à cet égard.

En 1941, un tableau classe aussi les natifs des États-Unis d'après leur période d'immigration au Canada. Ainsi, au Québec, 39\% sont arrivés avant 1911 et 42,5\% entre 1911 et $1930^{16}$. Cela témoigne de l'ancienneté du phénomène, même si une partie des personnes venues plus de trente ans auparavant sont déjà décédées ou ont déménagé.

Ces données sur le nombre d'immigrants américains vivant au Québec doivent être considérées comme une mesure minimale du phénomène. Elles ne fournissent qu'un instantané statique, tous les dix ans sans rendre compte de l'ampleur des mouvements migratoires ou des décès qui sont survenus entre deux recensements. Le nombre total de personnes originaires des États-Unis ayant vécu au Québec au cours de chaque décennie est évidemment supérieur à l'efectif constaté dans le recensement, sans qu'il soit possible de le mesurer avec précision. Ajoutons que ces données ne concement que les immigrants eux-mêmes et pas leurs enfants nés au Canada.

II faut toutefois s'interroger sur la proportion de ces Américains qui sont en fait des Franco-Américains, apparaissant dans les recensements comme étant à la fois nés aux États-Unis et d'origine ethnique française. Encore là, le recensement de 1941 est le premier à fournir cette information pour le Québec: leur part s'y établit à 68,4\% (34 336 personnes) et ils représentent $60,6 \%$ des 56640 Américains d'origine française recensés au Canada cette année-là'7. En 1931, la même information n'est donnée que pour l'ensemble du Canada: les effectifs sont alors de $55630^{18}$. Si

14. Recensement du Canada, 1941, IV, tableau 18. En 1931, les urbains comptent pour $76,5 \%$ des Américains de naissance, Recensement du Canada, 1931, I, tableau 80.

15. Recensement du Canada, 1921, II, tableau 38. Le même tableau fournit des données comparables pour 1911, mais elles ne portent que sur une douzaine de villes.

16. Recensement du Canada, 1941, IV, tableau 26.

17. Recensement du Canada, 1941, IV, tableau 20. Les données sur la langue maternelle vont dans le même sens: $66,2 \%$ des recensés québécois nés aux États-Unis déclarent le français (tableau 35).

18. Recensement du Canada, 1931, XIII, tableau 6. 
on estime la part du Québec en appliquant le rapport observé en 1941 $(60,6 \%)$, on obtient le chiffre de 33712 personnes, soit $68,2 \%$ de tous les Américains vivant au Québec, une proportion quasi identique à celle de 1941. Cette proportion peut-elle être appliquée rétroactivement? Étant donné la chronologie du mouvement d'émigation aux États-Unis, il est vraisemblable de penser que plus on recule dans le temps, au xix siècle, moins sera forte la proportion de Franco-Américains pami les immigrants nés aux États-Unis On pourrait le vérifier pour les recensements de 1871, 1881 et $1901^{19}$ en recourant non pas aux tableaux imprimés, muets sur le sujet, mais aux fiches individuelles remplies par les recenseurs; ce serait un travail d'une ampleur considérable.

II se pourrait qu'une partie des Américains d'origine française recensés au Québec en 1941 ne soient pas d'ascendance canadienne-fançaise proprement dite (c'est-à-dire issue du Québec ou de sa diaspora), mais qu'ils soient d'ascendance acadienne ou encore issus de familles européennes ayant d'abord émigré aux États-Unis. Le recensement ne permet pas de le savoir, mais nous pouvons faire l'hypothèse que l'impact de ce phénomène sur les résultats n'est pas considérable ${ }^{20}$. II faut plutôt mettre l'accent sur le constat principal: en 1931, comme en 1941, la majorité des immigrants nés aux États-Unis et vivant au Québec sont des Franco-Américains.

Les données sur le pays de naissance sont cependant insufisantes pour évaluer la présence franco-américaine au Québec Beaucoup de Canadiens français ayant émigré aux États-Unis sont ensuite revenus au Québec; aux yeux des recenseurs, ces personnes sont nées au Canada et sont d'origine française. Le recensement de 1941 posait toutefois une autre question, sur le dernier lieu de résidence permanent: 96160 recensés québécois ont répondu les États-Unis et, pami eux, 43774 étaient nés au Québec ${ }^{21}$. Ailleurs, le même recensement indique, parmi la

19. Rappelons que le recensement de 1891 ne contient pas de question sur l'origine ethnique.

20. Par exemple, en 1941, seulement 26 personnes nées aux États-Unis et résidant au Québec ont comme pays d'allégeance la France et 7 la Belgique. Recensement du Canada, 1941, IV, tableau 34; par ailleurs, les données du recensement ne permettent pas de distinguer les Acadiens des Canadiens français puisque tous sont d'origine ethnique française.

21. Ce dernier chiffre représente $89 \%$ de tous les natifs du Québec dont le dernier lieu de résidence était à l'étranger. Recensement du Canada, 1941, IV, tableau 24; Yolande Lavoie, «Les mouvements migratoires des Canadiens entre leur pays et les États-Unis au $\mathrm{xix}^{\mathrm{e}}$ et $\mathrm{au} \mathrm{xx}^{\mathrm{e}}$ siècles: étude quantitative», Hubert Charbonneau, dir., La population du Québec: études rétrospectives (Montréal, Boréal Express, 1973), 83. 
population immigrante du Québec, 48203 Canadiens rapatriés (ayant vécu un an ou plus à l'étranger), mais ne précise pas de quel pays ils viennent ${ }^{22}$. Dans l'un et l'autre cas, on ne sait pas l'origine ethnique de ces personnes, mais on peut estimer qu'elle est en majorité française. II semble donc qu'en 1941 il y a au Québec à peu près autant de Canadiens français natifs du Québec et rapatriés des États-Unis que d'immigrants américains d'origine française. Nous reviendrons plus loin sur la question des effectifs des Franco-Américains rentrés au pays.

Quant aux immigrants qui ne sont pas d'origine française, leurs effectifs comprennent probablement des descendants d'émigrants canadiens-anglais, puisque l'appel du sud a touché aussi bien les angophones que les francophones ${ }^{23}$. Les recensements ne permettent pas de les distinguer, car la plupart des Yankees sont eux aussi d'origine ethnique britannique. II faut donc conclure que les Américains proprement dits, sans liens antérieurs avec le Canada, n'étaient pas légion au Québec. Entre le début du $\mathrm{xix}^{\mathrm{e}}$ et le milieu du $\mathrm{xx}^{\mathrm{e}}$ siècle, leurs effectifs ont tout de même atteint au total quelques dizaines de milliers de personnes, sans compter leurs descendants, ce qui est suffisant pour s'y intéresser. Voyons donc ce que I'historiographie nous apprend à leur sujet.

\section{I es a méricains}

L'historiographie relative à la présence en sol québécois des Américains considérés collectivement, est concentrée dans le temps et dans l'espace: elle concerne surtout la première moitié du xix ${ }^{\mathrm{e}}$ siècle et se limite à des régions spécifiques. Pour les périodes subséquentes elle est beaucoup plus éclatée, dans des biographies d'individus et d'entreprises et dans quelques études sectorielles Nous essaierons d'en dégager une périodisation de l'immigration américaine au Québec et de mettre en lumière ses principales caractéristiques.

Même si nous commençons notre étude en 1792, il est utile de rappeler que, pendant les trois premières décennies qui suivent la Conquête

22. Recensement du Canada, 1941, IV, tableau 26. Si on utilisait le pourcentage calculé à la note 21 (89\%), on obtiendrait le chiffre de 42900.

23. Sur l'émigration des Canadiens anglais aux États-Unis voir, entre autres, Randy W illiam Widdis, W ith Scarcely a Ripple: Anglo-Canadian M igration into the United States and Western Canada, 1880-1920 (M ontréal, M cGill-Q ueen's University Press, 1998), 418 p. et Bruno Ramirez, Crossing the 49th Parallel: Emigration from Canada to the USA, 1900-1930 (Cornell University Press, à paraître). 
britannique, deux mouvements migratoires de faible amplitude en provenance du sud sont perceptibles au Québec. D'abord, parmi les marchands qui s'installent dans le sillage des amées d'occupation, un certain nombre sont nés ou ont séjoumé dans les Treize Colonies². Quelques-uns d'entre eux, tels Alexander Henry et Lucius Levy Solomons, se lancent dans la traite des fourrures et établissent leur quartier général à Montréa25. Les tensions croissantes entre l'Angleterre et ses établissements américains ainsi que les arantages de localisation de Montréal incitent en outre des marchands de fourrures de la colonie de New York, tels Robert Ellice ou Simon McTavish, à déménager dans cette ville ${ }^{26}$.

La seconde vague, un peu plus nombreuse, est celle des Loyalistes, ces réfugiés politiques qui ont dû quitter leur pays pendant ou immédiatement après la guerre d'Indépendance américaine. Les autorités britanniques les orientent systématiquement soit vers les colonies de l'Atlantique, soit vers le territoire du futur Haut-Canada, de sorte qu'on estime à environ 2000 le nombre de ceux qui s'établissent au Québec, surtout en Gaspésie et autour de Sore|27. Entre 1783 et 1792, un groupe de 500 à 600 personnes - des Loyalistes qui ne sont pas reconnus officiellement par les autorités et parmi lesquels se trouvent des individus d'origine allemande ou hollandaise - s'installent aussi autour de la baie Missisquoi, dans les seigneuries de Noyan, Foucault et Saint-Armand; plusieurs iront ensuite coloniser les Cantons de l'Es²8. Au total, l'arrivée des Loyalistes proprement dits n'a pas un impact considérable au Québec: phénomène très localisé, il pemet tout de même la naissance de nouvelles communautés rurales, en marge des principaux établisse-

24. Donald Creighton, The Empire of the St. Lawrence (Toronto, Macmillan, 1956), $22-25$.

25. David Arthur Armour, «Alexander Henry», Dictionnaire biographique du Canada (DBC), VI : 348-351; Walter S. Dunn Jr., «Lucius Levy Solomons», DBC, IV : 779-780.

26. James M. Colthart, «Robert Ellice», DBC, IV : 281-282; Fernand Ouellet, «Simon McTavish», DBC, V : 617-625.

27. Bruce G. Wilson, «Loyalists», The Canadian Encyclopedia (Edmonton, Hurtig, 1988), 2: 1250, deuxième édition; Ronald Rudin, Histoire du Q uébec anglophone (Québec, IQ RC, 1986), 52 55; Jules Bélanger et al., Histoire de la Gaspésie (M ontréal/ Q uébec, Boréal Express/ Institut québécois de recherche sur la culture, 1981), 144-145; J. M. Bumsted, "Americans», Paul Robert Magocsi, dir., Encyclopedia of Canada's Peoples (Toronto, University of Toronto Press, 1999), 187.

28. Jean-Pierre Kesteman, Peter Southam et Diane Saint-Pierre, Histoire des Cantons de I'Est (Q uébec, IQRC, 1998), 83-87; J. I. Little, Évolution ethnoculturelle et identité régionale des Cantons de I'Est (Ottawa, Société historique du Canada, coll. «Les groupes ethniques du Canada», n 13, 1989), 5-6. À noter que la seigneurie de Saint-Armand est incluse dans le territoire des Cantons de l'Est. 
ments francophones À long terme, si l'on tient compte de leur descendance, l'effet est plus marqué, mais il ne faut pas l'exagérer en confondant, comme on l'a fait trop souvent, ces réfugiés loyalistes et les autres Américains qui arrivent en grand nombre au cours des décennies suivantes.

À partir de la fin du xviII ${ }^{\mathrm{e}}$ siècle s'amorce une série de mouvements d'immigration en provenance des États-Unis II est possible d'y distinguer trois grandes périodes, chacune marquée par la prédominance, qui n'est pas exclusive, d'un type d'immigrants.

\section{Le colon, 1792-1820}

La vague migratoire américaine qui atteint les Cantons de l'Est à partir de la fin du xviII ${ }^{\text {e }}$ siècle est beaucoup plus importante et mieux connue que les précédentes L'ouverture de cette région à la colonisation, en 1792, en marque le point de départ. Les habitants des États de la Nouvelle-Angleterre trouvent là un exutoire à la pression démographique du monde rural, sans trop s'éloigner de leur milieu d'origine.

Au début, on s'inspire de modèles qui avaient cours dans les colonies américaines pour mettre au point le système du leader and associates. Des colons potentiels, les associés, se regroupent sous la direction d'un chef de file, le leader, et obtiennent la concession d'un ensembe de terres, parfois même d'un canton entier. Le leader doit faire arpenter les terres et en faciliter l'accès; il en reçoit une partie en compensation, tandis que les autres sont distribuées à chacun des associés Ce système permet de mobiliser des ressources plus importantes que dans un régime de colonisation individuelle. D'après G. F. McGuigan, cinquante groupes de leader and associates reçoivent ainsi d'importantes concessions entre 1792 et 1809, et amorcent le peuplement de la partie sud des Cantons de l'Est par des colons américains ${ }^{29}$.

Ce mouvement migratoire, une fois lancé, s'alimente régulièrement de nouveaux apports issus de la Nouvelle-Angleterre. Ralenti par la guerre de 1812-1814, puis par de mauvaises récoltes, il reprend dans les années 1820 et 1830, quoique l'ouverture du Midwest offre désormais une solution de rechange aux émigrants potentiels, attirant même des

29. G. F. McGuigan, «La concession des terres dans les Cantons de l'Est du Bas-Canada, 1763-1809», Recherches sociographiques, 4,1 (janvier-avril 1963): 71-89; id., "Administration of Land Policy and the Growth of Corporate Economic Organization in Lower Canada, 17911809», W. T. Easterbrook et M. H. Watkins, dir., Approaches to Canadian Economic History (Toronto, McClelland and Stewart, coll. «Carleton Library», n 31, 1967), 99-109. 
colons américains déjà établis dans les Cantons de l'Est. Depuis 1815, l'immigration en provenance des îles Britanniques contribue aussi au peuplement de la région, mais les Américains demeurent majoritaires Selon Jean-Pierre Kesteman, «en y incluant leurs descendants nés au Canada, on peut évaluer qu'en 1840 ils forment à peu près les deux tiers de la population totale des Cantons de l'Est et que cette propotion atteint près de $90 \%$ dans les cantons limitrophes à l'État du Vermont ${ }^{30} \%$.

On ne saurait exagérer l'importance de ce mouvement migratoire. Les Américains ont ouvert une région à la colonisation, ils y ont construit des chemins, défriché des terres, aménagé des moulins implanté des villages, développé un ensemble de services et d'institutions. Même si plusieurs ont ensuite quitté la région, leur présence a eu des effets durables tant sur le paysage que sur la société.

Le régime du leader and associates est également utilisé dans l'Outaouais. Le leader le plus en vue y est Philemon Wright qui s'établit à Hull en 1800 avec quelques familles venues, comme lui, du Massachusetts. II fait de la colonisation agricole, mais il devient aussi un homme d'affaires important, se lançant dans l'exploitation forestière et dans diverses autres activités. La famille Wright occupe une place de premier plan en Outaouais pendant la première moitié du $\mathrm{xIx}^{\mathrm{e}}$ siècle. Elle ne réussit pas cependant à attirer un grand nombre d'immigrants américains et le peuplement de la région se fait surtout par des colons britanniques et canadiens-français ${ }^{31}$.

\section{L'entrepreneur, 1820-1880}

Ainsi, au début du $\mathrm{xxx}^{\mathrm{e}}$ siècle, la figure de l'immigrant américain qui prédomine au Québec est celle du colon. À partir des années 1820, et pour plus d'un demi-siècle, lui succède celle de l'immigant entrepreneur, qu'on appellerait aujourd'hui immigrant investisseur. Disposant d'un certain capital et d'un savoir technique acquis aux États-Unis il s'installe au Québec pour y mettre sur pied une entreprise nouvelle, autre qu'une exploitation agricole.

Le phénomène est visible dans les Cantons de l'Est où, selon Kesteman, les nouveaux arrivants américains sont moins souvent des colons et «plus fréquemment des artisans, des professionnels et des entrepre-

30. Jean-Pierre Kesteman et al., H istoire des Cantons del'Est, 114; voir aussi J. I. Little, Évolution ethnoculturelle... 8, 13-17.

31. Chad Gaffield, dir., Histoire de l'O utaouais (Québec, IQRC, 1994), 121-156; Fernand Ouellet et Benoît Thériault, «Philemon W right», DBC, VII : 1003-1007. 
neurs ${ }^{32} \gg$. Certes, avant eux, il y avait déjà des hommes d'affaires, notamment parmi les leaders qui se sont souvent réservés les terrains bordant les chutes où ils ont fait aménager des moulins. En outre, parmi les fils des colons de la période précédente, nombreux sont ceux qui, tout en étant nés au Québec, séjournent en Nouvelle-Angleterre pour y poursuivre leurs études ou pour y acquérir une formation technique. Après 1820, les nouveaux immigrants américains et les descendants de leurs prédécesseurs - qu'ils soient grands propriétaires fonciers, marchands ou simples artisans - jouent encore une fois un rôle fondamental dans le développement économique de la partie méridionale des Cantons de I'Est. Ils sont les principaux responsables de la première phase d'industrialisation régionale qui s'appuie à la fois sur du capital endogène et sur le transfert de technologie ou de savoir-faire en provenance de la Nouvelle-Angleterre ${ }^{33}$.

La présence américaine est aussi notable à Montréal. Tout en reconnaissant la difficulté de mesurer avec précision leurs effectifs ou leur influence, Gerald Tulchinsky estime qu'après les Écossais, les hommes d'affaires nés aux États-Unis «comprised probably the second most significant national group in the economic life of Montreal in the period from 1837 to $1853^{34} \gg$. Ils sont notamment actifs dans le commerce des denrées - légal aussi bien qu'illégal - avec les États limitrophes, ceux de New York et du Vermont en particulier. L'un d'eux, Horatio Gates, natif du Massachusetts, est le chef de file de la communauté américaine de la ville. Actif dans plusieurs secteurs de l'économie montréalaise, dont la banque, il est nommé au Conseil législatif du Bas-Canada ${ }^{35}$. Un autre, Harrison Stephens, originaire du Vermont, est aussi I'un des grands propriétaires fonciers de la ville au milieu du siècle $e^{36}$.

Les Américains dominent aussi le secteur de la quincaillerie dans lequel s'illustrent, entre autres, Jacob De Witt et John Frottingham. Le premier, né au Connecticut, est surtout connu comme cofondateur en 1835 de la Banque du Peuple (d'abord Viger, DeWitt et Compagnie) dont il sera un des dirigeants jusqu'à sa mort, en 1859; de plus, il est actif

32. Jean-Pierre Kesteman et al., Histoire des Cantons de l'Est, 114.

33. Jean-Pierre Kesteman, Une bourgeoisie et son espace: industrialisation et développement du capitalisme dans le district de Saint-François (Québec), 1823-1879, thèse de doctorat (histoire), Université du Québec à M ontréal, 1985, 847 p.

34. Gerald J. J. Tulchinsky, The River Barons: M ontreal Businessmen and the Growth of Industry and Transportation, 1837-53 (Toronto, University of Toronto Press, 1977), 11.

35. Jean-Claude Robert, «H oratio Gates», DBC, VI: 304-308.

36. Gerald J. J. Tulchinsky, «H arrison Stephens», DBC, XI : 942-943. 
en politique, étant élu député à plusieurs reprises ${ }^{37}$. Le second, originaire du Maine, met sur pied, avec William Workman, ce qui devient «la plus importante entreprise de commerce de gros en quincaillerie et en ferronnerie de l'Amérique du N ord britannique ${ }^{38} \gg$. De nombreux autres Américains sont présents dans diverses branches de l'activité commerciale. On relève aussi des financiers, tel John E. Mills, du Massachusetts, maire de Montréal en 1847 et qui, après s'être dévoué auprès des victimes de l'épidémie de typhus qui sévit cette année-là, est lui-même emporté par la maladie ${ }^{39}$.

II faut aussi souligner la contribution d'artisans et d'entrepreneurs américains à la naissance de l'industrie marufacturière de la ville. Ainsi, les frères Ward créent Eagle Foundry en 1819 et sont pendant deux décennies les plus importants fabricants de moteurs de navires. Ira Gould, I'un des premiers à utiliser le potentiel énergétique du canal de Lachine, fait ériger une grande meunerie en 1846. La famille Lyman domine la fabrication de produits chimiques et phamaceutiques ${ }^{40}$.

$\mathrm{Au} \mathrm{xix}^{\mathrm{e}}$ siècle, la fabrication de chaussures, héritière d'une longue tradition artisanale, occupe une place exceptionnelle à Montréal. Là encore, des Américains jouent un rôle clé en y développant la production mécanisée, comme l'a montré joanne Burgess. Dès 1849, Champion Brown et William Sullivan Childs, originaires du Massachusetts - alors le principal centre de la chaussure aux États-Unis -, introduisent une première machine dans leur établissement; d'autres suivront par la suite et permettront à Brown $\&$ Childs de se hisser à la tête de cette industrie ${ }^{41}$. Celle-ci attirera également à Montréal d'autres entrepreneurs originaires des États-Unis, dont Evan Fisher Ames, W. A. Dunham et D. Kieley 42 .

À l'extérieur de Montréal et des Cantons de l'Est, des entrepreneurs américains sont à l'œuvre dans diverses régions. L'industrie forestière en attire un certain nombre au milieu du siècle, notamment dans I'Outaouais où s'installe entre autres Ezra Butler Eddy, dont les usines

37. Jean-Claude Robert, «Jacob De Witt», DBC, VIII : 243-245.

38. Gerald J. J. Tulchinsky, «John Frothingham», DBC, IX: 317-318.

39. Claude-V. Marsolais, Luc Desrochers et Robert Comeau, Histoire des maires de M ontréal (M ontréal, VLB, 1993), 44-49.

40. Gerald J. J. Tulchinsky, The River Barons... , 203-231; Frederick H. Armstrong, «Benjamin Lyman», DBC, X: 493-494.

41. Joanne Burgess, «L'industrie de la chaussure à Montréal, 1840-1870 - Le passage de I'artisanat à la fabrique», RHAF, 31,2 (septembre 1977): 187-210.

42. M ontreal Illustrated 1894 (M ontréal, Consolidated Illustrating, 1894), 113, 200, 207. 
occupent une place considérable dans l'économie de Hull, et qui sera maire de cette ville et député à Québec ${ }^{43}$. En Mauricie, Norcross et Philips font construire une grande scierie, appelée le «moulin des Américains», mais leur aventure est de courte durée ${ }^{44}$. Quelques décennies plus tard, c'est la construction ferroviaire qui attire un Horace Jansen Beemer, fort actif à Québec et au Lac Saint-Jean ${ }^{45}$. Malgré tout, les Américains sont peu nombreux dans ces régions. Ainsi, François Guérard n'en relève que 2 parmi 173 notables de Trois-Rivières dans le dernier tiers du $\mathrm{xix}^{\mathrm{e}}$ siècle: Thomas G. Farmer, hôtelier, et son fils, Fred F. Farmer, homme d'affaires et consul de son pays d'origine ${ }^{46}$.

L'immigration en provenance des États-Unis à cette époque n'amène pas que des hommes d'affaires. Ces derniers font parfois venir des artisans ou des ouvriers qualifiés, comme c'est le cas chez Eagle Foundry ${ }^{47}$. II vient aussi des pasteurs, des médecins, des avocats et des membres d'autres professions libérales. Charles Dewey Day n'est peut-être pas le plus représentatif parce qu'il est encore enfant quand ses parents quittent le Vermont et qu'il fait ses études à Montréal, mais il a une influence considérable dans la société québécoise du milieu du $\mathrm{xix}^{\mathrm{e}}$ siècle. Férocement opposé aux Patriotes, il réclame, en tant que procureur public, la pendaison de plusieurs rebelles. Grand avocat d'affaires, ministre, juge, chancelier de I'Université McGill, il est en outre un des trois commissaires responsables de la rédaction du nouveau Code civil du Bas-Canada ${ }^{48}$. Henry D. Lawrence, né lui aussi au Vermont et formé en Nouvelle-Angleterre, devient principal de la Sherbrooke Academy en 1873, puis avocat à Sherbrooke à partir de $1883^{49}$.

Ces quelques exemples suffisent à montrer qu'au $x{ }^{e}$ siècle, les immigrants américains ont un poids significatif dans certaines régions et

43. Jean Hamelin et Yves Roby, Histoire économique du Q uébec, 1851-1896 (Montréal, Fides, 1971), 218; Chad Gaffield, dir., Histoire de l'O utaouais, 269-275; Henry James Morgan, The Canadian Men and Women of the Time (Toronto, William Briggs, 1898), 302-303.

44. René Hardy et Normand Séguin, Forêt et société en Mauricie(M ontréal, Boréal, 1984), 183.

45. Jean Benoit, «H orace Jansen Beemer», DBC, XIV : 54-56.

46. Communication personnelle de l'auteur; François Guérard, «Les notables trifluviens au dernier tiers du $\mathrm{xix}^{\mathrm{e}}$ siècle: stratégies matrimoniales et pratiques distinctives dans un contexte d'urbanisation», RHAF, 42,1 (été 1988) : 27-46.

47. Gerald J. J. Tulchinsky, The River Barons..., 211-215.

48. Carman Miller, «Charles Dewey Day», DBC, XI: 261-263; Brian Young, The Politics of Codification: The Lower Canadian Civil Code of 1866 (M ontréal, M cGill-Queen's University Press, 1994), 84-95.

49. J. Castell Hopkins, The Canadian Album. Encyclopedic Canada or the Progress of a Nation (Brantford/ Toronto, Bradley-Garetson, 1896), V : 291 (numérotée 305 par erreur). 
contribuent au développement économique et social du Québec De plus, ils ouvrent des temples et des écoles et jouent un rôle clé dans l'essor du mouvement de tempérance ${ }^{50}$.

II reste cependant beaucoup à faire pour comprendre les modalités d'insertion de ces nouveaux venus dans le tissu social québécois On en connaît certains éléments grâce aux travaux de Kesteman et de Little sur les Cantons de l'Est, et à ceux de Tulchinsky sur Montréal, probablement les deux seules régions du Québec où les Américains sont suffisamment nombreux pour former une communauté distincte.

Pendant les premières décennies de formation des Cantons de l'Est, une lutte sourde oppose les pionniers américains, héritiers d'une culture relativement égalitaire, aux représentants du pouvoir britannique, nettement plus élitistes et autoritaires. Les premiers ont une longue tradition de démocratie locale et de gestion de leurs propres affaires, tandis que les seconds cherchent à imposer un régime hiérarchique dont les décisions sont centralisées dans les officines du gouvernement colonial et relayées par les fonctionnaires locaux. Les différences se reflètent aussi dans les appartenances religieuses, les Américains ayant tendance à préférer les confessions identifiées au courant évangélique. Avec le temps, plusieurs notables et hommes d'affaires d'ascendance américaine voient qu'ils ont avantage à se rapprocher des représentants de l'ordre établi. Dès lors, les clivages ne sont plus seulement liés aux orignes, mais ont aussi une dimension socio-économique et, dans les années 1830, la population est divisée entre réformistes et constitutionnalistes Les premiers font d'abord des gains politiques, surtout parmi les colons américains, mais l'agitation qui conduit aux Rébellions fait basculer une bonne partie de leurs partisans qui craignent l'emprise politique des Canadiens. Ainsi, le loyalisme britannique triomphe dans la région, même chez les Américains, malgré le bref épisode annexionniste de $1849^{51}$.

Dans la seconde moitié du siècle, la différence américaine ne semble plus être un facteur fondamental d'identification dans les Cantons de I'Est. L'historiographie accorde en effet beaucoup plus d'importance à la

50. Voir l'exemple du pasteur Ammi Parker: J. I. Little, «Serving "the N orth East Corner of the Creation": The Community Role of a Rural Clergyman in the Eastern Townships of Quebec, 1829-1870», Histoire sociale/ Social History, 30,59 (mai 1997): 21-53.

51. Jean-Pierre Kesteman et al., Histoire des Cantons de l'Est, 171-217; J. I. Little, State and Society in Transition: The Politics of Institutional Reform in the Eastern Townships, 1838-1852 (M ontréal, McGill-Queen's University Press, 1997), 7-47. 
distinction entre la population anglo-protestante ou anglophone, prise globalement, et la population canadienne-française qui «envahit»la région à partir des années 1840 . II semble donc y avoir un certain amalgame de la population anglophone au fur et à mesure que les éléments nés au Canada dépassent en nombre les immigrants ${ }^{52}$. L'héritage culturel et politique américain continue tout de même à façonner cet amal game.

A Montréal, les immigrants américains forment aussi une communauté distincte parmi les anglophones de la ville pendant les premières décennies du $\mathrm{xIx}^{\mathrm{e}}$ siècle. Selon Tulchinsky, ils sont souvent victimes de discrimination, du moins au début, et ils s'intègrent peu à la vie sociale des Anglo-Écossais et à la vie politique municipale ou provinciale. Un événement révélateur est leur décision, en 1822, de quitter la congégation presbytérienne St. Andrew, où ils ne se sentent pas à l'aise, pour créer I'American Presbyterian Congregation, qui disposera de son propre temple à partir de 1826. Est-ce un sentiment de rejet partagé qui incite certains entrepreneurs américains à s'associer à des Canadiens français dans les années 1830 pour fonder la City Bank et la Banque du Peuple? Quoi qu'il en soit, la majorité d'entre eux saisissent aussi toutes les occasions de bonnes affaires avec les Anglo-Écossais. Leur force réside toutefois dans le réseau de relations qu'ils ont su développer ou conserver aux États-Unis ${ }^{33}$.

\section{Le gestionnaire et l'expert, 1880-1940}

À compter des années 1880, I'entrepreneur individuel continue à manifester sa présence mais de plus en plus, il cède la place au gestionnaire et à l'expert comme figures emblématiques de l'immigrant américain au Québec. Certains ne viennent que pour un temps limité, la durée d'un mandat ou d'un contrat, d'autres s'installent pour longtemps et s'intègrent dans la société d'accueil. Ce changement dans le type d'immigration s'explique à la fois par l'émergence de la grande entreprise et par l'avance économique et technologique dont jouissent les États-Unis par rapport au Canada, surtout après la guerre de Sécession.

Les hommes d'affaires canadiens qui mettent sur pied une nouvelle entreprise peuvent continuer à faire appel à du personnel qualifié et expérimenté venant de Grande-Bretagne, mais parfois ils préfèrent profiter de l'avance des États-Unis et y recruter les ressources humaines

52. J. I. Little, Évolution ethnoculturelle..., 34.

53. Gerald J. J. Tulchinsky, The River Barons..., 10-13; id., «John Frothingham», 317-318; Newton Bosworth, Hochelaga Depicta (M ontréal, William Greig, 1839), 113-114. 
dont ils ont besoin, compte tenu des différences qui existent entre les contextes européen et nord-américain. C'est une tendance notoire dans les entreprises ferroviaires. William Cornelius Van Horne en fournit une excellente illustration. Natif de l'Illinois, il a déjà près de vingt-cing ans d'expérience dans les chemins de fer américains quand le Canadien Pacifique lui confie la direction générale en 1882, puis la présidence, de 1888 à 1899; il devient alors l'un des membres les plus en vue de la bourgeoisie montréalaise ${ }^{54}$. En 1882, Van Horne recrute un autre Américain, Thomas George Shaughnessy, qui lui succèdera à la présidence, de 1899 à 191855. Plusieurs autres experts ferroviaires des États-Unis, dont Isaac Gouverneur Ogden, travaillent aussi pour l'entreprise pendant ses premières décennies d'existence ${ }^{56}$. Même la très londonienne compagnie du Grand-Tronc cède à l'appel du sud en embauchant comme directeur général, en 1896, un administrateur ferroviaire expérimenté, Charles M elville Hays, natif de l'Illinois, afin qu'il réorganise le réseau selon des méthodes américaines ${ }^{7}$. Après l'étatisation du Grand Tronc, le gouvernement fédéral fait à son tour appel à un dirigeant ferroviaire américain qui a en outre travaillé en Grande-Bretagne, H enry Worth Thornton, pour devenir président du Canadien NationaF8.

L'immigration de gestionnaires est encore plus associée à l'expansion des grandes entreprises américaines Afin d'étendre leur marché et de contourner les barrières tarifaires, celles-ci créent des filiales au Canada où leurs concurrentes locales sont soit inexistantes, soit beaucoup plus faibles qu'elles II en résulte un important apport de capitaux et de technologie américains, utilisés dans le but de répondre aux objectifs du siège social ; les gestionnaires et les techniciens envoyés des États-Unis semblent être de simples exécutants, mais ils disposent parfois d'une certaine marge de manœuvre pour s'adapter aux conditions locales ${ }^{59}$. M ême si, au xix ${ }^{\mathrm{e}}$ siècle, Montréal accueille le siège canadien d'un certain nombre d'entreprises américaines, il faut préciser qu'à long terme, le

54. Theodore A. Regehr, «William Cornelius Van Horne», DBC, XIV : 1124-1131.

55. John A. Eagle, «Thomas George Shaughnessy », The Canadian Encyclopedia, 3: 1988.

56. David D. Harvey, Americans in Canada, 160-161; Henry James Morgan, The Canadian Men..., 780.

57. Theodore A. Regehr, «Charles Melville Hays», DBC, XIV : 503-505.

58. John A. Eagle, «Sir Henry W orth Thornton», The Canadian Encyclopedia, 4: 2154.

59. Voir par exemple Graham D. Taylor, «Charles F. Sise, Bell Canada and the Americans: A Study of Managerial Autonomy, 1880-1905», Douglass McCalla, dir., The Development of Canadian Capitalism: Essays in Business History (Toronto, Copp Clark Pitman, 1990), 145-166. 
Québec n'est pas le principal récipiendaire de ces mouvements de capitaux et de personnes puisque, très tôt, ces entreprises concentrent leurs investissements canadiens en Ontario. Les données sur le secteur manufacturier montrent que, déjà en 1913, Montréal ne compte que $12 \%$ des filiales américaines au Canada, tandis que Toronto en a 21\%. En 1936, l'écart s'est creusé: le Québec n'a que 16\% du total, dont 13\% pour Montréal, tandis que I'Ontario en rafle $66 \%$, dont $32 \%$ pour Toronto. En 1961, cette dernière en compte presque sept fois plus que M ontréal60.

Parmi les gestionnaires installés dans la métropole, Charles Fleetwood Sise, natif du New Hampshire, ressort nettement. En 1880, la compagnie National Bell Telephone, de Boston, l'envoie à Montréal pour organiser sa filiale canadienne. II devient le véritable fondateur de Bell Canada qu'il dirige d'une main de fer pendant 35 ans. II meurt à Montréal en 1918 et son fils aîné sera à son tour président de l'entreprise de 1925 à 1944².

C'est cependant dans l'exploitation des ressources naturelles que la présence américaine se manifeste avec le plus d'ampleur au Québec pendant les premières décennies du $\mathrm{xx}^{\mathrm{e}}$ siècle. L'industrie de l'aluminium en fournit la meilleure illustration. En 1899, la société Pittsburgh Reduction (ancêtre d'Alcoa) décide d'aménager sa première usine canadienne à Shawinigan. Au moins jusqu'en 1938, celle-ci sera dirigée par des Américains délégués par le siège social; il en sera longtemps de même pour les ingénieurs et pour plusieurs cadres, les premiers ingénieurs canadiens n'étant embauchés qu'à partir des années 1920. Une situation sembable se produit lors de l'installation d'Alcoa à Arvida: la plupart des principaux cadres de I'usine sont américains; selon Duncan C. Campbell, ils sont plus d'une cinquantaine entre 1926 et 1928. Quand, en 1928, Alcoa décide d'accorder son indépendance à sa filiale canadienne et de lui rattacher toutes ses activités internationales, les hauts dirigeants de la nouvelle société Aluminium Limitée (derenue Alcan) proviennent tous d'Alcoa et sont tous d'origine américaine; en fait, son président est le

60. Gerald Bloomfield et al., «Évolution de la structure manufacturière», Donald Kerr et Deryck W. Holdsworth, dir., Atlas historique du Canada. III. Jusqu'au coeur du xxe siècle (Montréal, Presses de I'Université de Montréal, 1990), pl. 7; Herbert Marshall, Frank Southard et Kenneth W. Taylor, Canadian-American Industry (Toronto, McClelland and Stewart, 1976), 220222; D onald Kerr et Gerald Bloomfield, «La continuité des caractéristiques manufacturières», D. Kerr et D. W. Holdsworth, Atlas historique du Canada, III, pl. 51.

61. Robert E. Babe, "Charles Fleetwood Sise», DBC, XIV : 1016-1019; Graham D. Taylor, «Charles F. Sise, Bell Canada and the Americans... », 145-166. 
propre frère du président de l'ancien siège socia². Par la suite, on assistera à une canadianisation progressive, mais il faut reconnaître que, pendant son premier demi-siècle d'existence au Québec, l'industrie de l'aluminium a été fortement redevable, non seulement de la technologie et du capital américains, mais aussi d'un personnel clé originaire des ÉtatsUnis.

Dans les secteurs de l'hydro-électricité et des pâtes et papiers, on note la présence au Québec de filiales de sociétés américaines - celles d'Union Bag ou d'International Paper, par exemple - , mais plusieurs entreprises ont une existence autonome. Parmi celles-ci, notamment dans le cas de Shawinigan Water and Power et de Laurentide Paper, les Américains jouent souvent un rôle décisif à la fois dans le financement initial et dans la gestion. Étudiant la Mauricie, Claude Bellaance a calculé que $22 \%$ des administrateurs et cadres supérieurs des grandes entreprises de la région sont des Américains en 1911; leur part grimpe à $35 \%$ en 1930 et est encore de $32 \%$ en $1950^{63}$. Deux d'entre eux ont un rôle fondamental pendant les premières décennies d'existence de Shawinigan Water and Power: J. E. Aldred, financier de Boston ${ }^{64}$, et l'ingénieur Julian Cleveland Smith, natif de l'État de New York ${ }^{65}$. À la papetière Laurentide, George Chahoon Jr., aussi originaire de l'État de New York et arrivé en 1902, est l'âme dirigeante de la compagnie jusqu'à son intégration à Canada Power and Paper en $1928^{66}$.

Comme l'a montré Claude Bellarance, ces dirigeants américains ne limitent pas leur rôle au développement de leur entreprise et, au moins jusqu'en 1930, ils se préoccupent du développement social de la collectivité où elle est implantée. Ils interviennent de multiples façons dans l'aménagement et la gestion des villes de Grand-M ère et de Shawinigan ainsi que dans la mise en place de services publics, comme ce sera aussi le cas plus tard à Arvida. Cette stratégie relève d'une forme de paternalisme d'entreprise, mais elle exprime aussi une conception de la

62. Duncan C. Campbell, Mission mondiale Histoire d'Alcan. I: Jusqu'à 1950 (s.l., Ontario Publishing, 1985), 20-25, 75-76, 119-123.

63. Claude Bellavance, «Patronat et entreprise au $x^{e}$ siècle: l'exemple mauricien», RHAF, 38,2 (automne 1984): 188.

64. John H. Dales, Hydroelectricity and Industrial Development: Quebec 1898-1940 (Cambrige, Mass., Harvard University Press, 1957), 52, 64-65, 229.

65. Claude Bellavance, «Patronat et entreprise...», 190; Canadian Newspaper Service, Reference Book 1931-1932 (M ontréal, Canadian Newspaper Service, 1931), 384-385.

66. Jorge N iosi, «La Laurentide (1887-1928): pionnière du papier journal au Canada», RHAF, 29,3 (décembre 1975): 375-415. 
responsabilité sociale de l'entrepreneur. Chahoon, par exemple, s'identifie totalement à Grand-M ère, qu'il habitera jusqu'à sa mort, en 1951. De son côté, Aldred est le principal promoteur de l'école technique de Shawinigan qu'il contribue personnellement à financer ${ }^{67}$.

Ce sont là les personnages les plus célèbres, mais il y en a des dizaines d'autres qui, comme eux, poursuivent au Québec une carrière amorcée aux États-Unis et vivent dans la province pendant une longue période, souvent jusqu'à leur décès. Ces grands gestionnaires ne sont pas les seuls immigrants américains pendant cette période. Pour les besoins de leurs entreprises, qu'elles soient canadiennes ou américaines les dirigeants font venir des États-Unis plusieurs experts en divers domaines: des ingénieurs, des chimistes, des comptables, des vendeurs, des techniciens, etc. Sauf si l'un d'entre eux, tel Julian Cleveland Smith, atteint une certaine notoriété en se hissant aux plus hauts échelons la plupart restent relativement inconnus et n'ont pas fait l'objet d'études spécifiques. Combien sont-ils, quels sont leurs rôles, la durée de leur séjour au Québec? Ces questions ne sont guère abordées dans l'historiographie et il est impossible pour l'instant d'établir un portrait d'ensemble de ces experts.

Un groupe a suscité des recherches plus poussées, celui des architectes américains à l'œuvre à M ontréal entre 1880 et 1930. L'un des plus connus est Bruce Price à qui Van Horne confie la conception de la gare Windsor et de la gare-hôtel Viger à Montréal, ainsi que du Château Frontenac à Québec. II y en a cependant beaucoup plus comme le montrent les travaux d'Isabelle Gournay et de France Vanlaethem. En effet, de nombreuses agences américaines obtiennent des contrats à Montréal, non seulement de la part de leurs compatriotes, mais aussi d'entrepreneurs canadiens. Parmi elles on relève des noms tels M cKim, Mead and W hite, Babb, Cook and Willard, Warren and Wetmore, Carrère and H astings, Stevens and Lee, Thomas Lamb et de nombreux autres. Elles construisent des immeubles de banques ou de chemins de fer, des tours à bureaux, des hôtels et des cinémas Elles contribuent à transformer le paysage architectural de Montréal, jusque-là dominé par les modèles britanniques Leurs architectes ne séjournent pas longtemps au Québec, mais ils influencent la scène locale de multiples façons.

67. Claude Bellavance, Le patronat de la grande entreprise en M auricie, 1900-1950, mémoire de maîtrise (études québécoises), Université du Québec à Trois-Rivières, 1983, 45-64; voir aussi Robert Fortier, dir., Villes industrielles planifiées (M ontréal, CCA/ Boréal, 1996), 320 p 
D'une part, des agences montréalaises leur sont souvent associées pour la gestion des chantiers; d'autre part, un nombre croissant d'architectes montréalais, à l'exemple d'Edward Maxwell, vont faire un stage chez leurs confrères américains. En outre, quelques architectes américains dont H oward C. Stone, David Jerome Spence, Hugh G. Jones et Ernest Isbell Barott - décident d'immigrer à Montréal, compte tenu de l'intense activité de construction qui $s^{\prime} y$ déroul $e^{68}$. On peut y ajouter l'exemple de l'architecte paysagiste Frederick G. Todd, du New H ampshire, qui s'installe à M ontréal en 1900 et qui conçoit de nombreux plans d'aménagement au Québec, dont ceux de la ville de Mont-Royal, du parc de l'île Sainte-H élène et du parc des Champs de bataille $e^{69}$.

II faudrait des enquêtes semblables sur d'autres groupes professionnels, notamment les ingénieurs, les médecins et les scientifiques. II faudrait en savoir plus sur la présence des immigrants américains dans I'enseignement universitaire. En 1883, David Pierce Penhallow, natif du Maine, est embauché à McGill pour enseigner la botanique et il y contribuera au développement de cette discipline $e^{70}$. Y en a-t-il d'autres comme lui à la même époque? À quel moment se manifeste l'américanisation du corps professoral dans cette université qui pendant longtemps recrute surtout en Grande-Bretagne? Les questions à examiner restent nombreuses.

Par ailleurs, un autre type d'immigration, d'origine afro-américaine, se manifeste à M ontréal à la fin du xix siècle. Elle est liée à l'apparition des wagons-lits dans les trains. Le travail de porter, d'employé au service des voyageurs, y devient réservé aux Noirs dans les entreprises ferroviaires américaines, dont certaines desservent la métropole, et leurs homologues canadiennes emboîtent le pas Des Noirs des États-Unis qui occupent ces emplois en viennent à adopter Montréal comme pott d'attache et lieu de résidence. D'autres sont embauchés dans les gares comme Red Caps, porteurs de bagages. Ces immigrants forment le premier noyau de la communauté noire montréalaise, d'abord regroupée à proximité des deux grandes gares et qui s'étend ensuite au sud-

68. Isabelle Gournay et France Vanlaethem, dir., M ontréal métropole, 1880-1930 (Montréal, CCA/ Boréal, 1998), 112-114, 127-145; voir aussi M adeleine Forget, «La contribution américaine dans l'aménagement et l'architecture de Montréal: le gratte-ciel », Gérard Bouchard et Yvan Lamonde, dir., Québécois et Américains..., 319-337.

69. The Canadian W ho's W ho, II (1936-1937): 1073.

70. Henry James Morgan, The Canadian Men..., 813; W. Stewart Wallace, The Macmillan Dictionnary..., 657. 
ouest, vers Sainte-Cunégonde et Saint-H enri. Leurs effectifs sont ensuite grossis par l'apport de compatriotes qui viennent travailler dans les usines de guerre, pendant le premier conflit mondial, puis par d'autres qui, fuyant la prohibition, implantent des cabarets qui atteignent une certaine notoriété. Le gouvernement canadien met toutefois des entraves à l'immigration des Noirs, de sorte que le nombre des nouveaux venus est limité. Les difficultés économiques du début des années 1920 entraînent des départs de la métropole, mais au cours de cette décennie, les Américains forment encore près de la moitié de la communauté noire de Montréa ${ }^{71}$.

De simples ouvriers ou employés américains, d'origine autre qu'africaine, se sont-ils établis au Québec pendant cette période? C'est fort possible, compte tenu des besoins de main-d'œuvre des entreprises, à Montréal comme dans les nouvelles villes des régions de ressources. L'historiographie n'en a toutefois pas gardé la trace. On peut se demander également si des syndicalistes américains ont joué un rôle diect dans l'expansion des Unions internationales au Québec, à partir des années 1880. Robert Babcock a montré que l'American Federation of Labor et son président Samuel Gompers mettent beaucoup de temps arant de prendre conscience des exigences linguistiques de l'action syndicale dans la province ${ }^{72}$. Q u'en est-il des Unions elles-mêmes? Ont-elles délégué des organisateurs américains ou se sont-elles uniquement appuyées sur des Canadiens? En 1934, I'Union internationale des ouvriers du vêtement pour dames envoie à M ontréal Bernard Shane, natif du N ew Jersey, pour implanter l'organisation dans la métropole; il ne devait rester que quelques semaines, mais il s'y établira à demeure. Deux ans plus tard, on lui envoie en renfort une organisatrice talentueuse, Rose Pesotta, née en Ukraine et qui a immigré aux États-Unis en 1913. Ensemble, ils réussissent à syndiquer les midinettes francophones et dirigent la célèbre «grève de la guenille» de $1937^{73}$. Y a-t-il d'autres Américains qui connaissent au Québec une trajectoire semblable?

71. Dorothy W. Williams, Blacks in M ontreal, 1628-1986: An Urban Demography (Cowansville, Yvon Blais, 1989), 21-31; Robin W. Winks, The Blacks in Canada: A History (Montréal, M cGillQueen's University Press, 1997), 332-334, $2^{\mathrm{e}}$ édition.

72. Robert Babcock, «Samuel Gompers et les travailleurs québécois, 1900-1914», Fernand Harvey, dir., Le mouvement ouvrier au Québec (M ontréal, Boréal Express, 1980), 131-149.

73. Evelyn Dumas, Dans le sommeil de nos 0s. Quelques grèves au Québec de 1934 à 1944 (Montréal, Leméac, 1971), 55-72; Gemma Gagnon, «Rose Pesotta (1896-1965)», Maryse Darsigny et al., dir., Ces femmes qui ont bâti M ontréal (M ontréal, Remue-ménage, 1994), 225-227. 
Pour l'instant, malgré la présence de simples travailleurs, le gestionnaire et l'expert paraissent être l'élément le plus représentatif de l'immigration non francophone en provenance des États-Unis pendant la période qui va de 1880 à 1940 et même au-delà. Les tensions qui étaient apparues, au début du xix ${ }^{\mathrm{e}}$ siècle, entre immigrants américains et britanniques semblent s'être estompées. Certes, l'établissement d'entreprises américaines suscite des réactions négatives, mais celles-ci ne semblent pas s'étendre aux individus qui immigrent. L'historiographie parait les confondre désormais avec la masse de la population angophone. Dans leurs rapports avec les francophones, surtout avec leurs ouvriers et employés, ils sont plus perçus comme patrons ou comme angophones que comme Américains Ainsi, les études sur les villes nouvelles, telles Shawinigan ou Arvida, insistent plus sur la séparation territoriale des quartiers réservés aux cadres anglophones que sur les origines géographiques de ces derniers. Manifestement, l'insertion sociale et culturelle des immigrants américains après 1880 est une question qui mériterait d'être explorée.

\section{les franco-américains}

Entre 1840 et 1930, des centaines de milliers de Canadiens français du Québec émigrent, de façon permanente ou temporaire, aux États-Unis Les effets de ce mouvement migratoire sur le Québec même sont considérables. II faut d'abord évoquer les pertes humaines et matérielles Le départ d'un si grand nombre de personnes a en effet ralenti la croissance démographique ${ }^{74}$. II a peut-être contribué à réduire le poids des francophones au Québec et au Canada, mais on ne peut en ête certain, car l'émigration a aussi touché fortement les anglophones. II a freiné le développement de paroisses et même de régons entières, tout en faisant porter au nombre réduit des gens qui restaient le fardeau de soutenir les institutions locales Les migrants ont quitté avec des biens et un peu d'argent. Ils ont souvent, comme le suggère Bruno Ramirez, financé leur installation dans leur nouveau pays en utilisant leurs terres comme levier; éventuellement, une fois le départ devenu définitif, le produit de la vente de ces propriétés foncières a pu être rapatrié aux États-Unis ${ }^{75}$.

74. Yolande Lavoie, «Québécois et francophones dans le courant migratoire vers les ÉtatsUnis aux $\mathrm{xIx}^{\mathrm{e}}$ et $\mathrm{xx}^{\mathrm{e}}$ siècles», Critère, 27 (printemps 1980) : 205-219.

75. Bruno Ramirez, Par monts et par vaux. M igrants canadiens-français et italiens dans l'économie nord-atlantique, 1860-1914 (Montréal, Boréal, 1991), 50-56. 
Pour chaque famille, les possessions en cause sont assez modestes mais à l'échelle nationale, cela peut représenter un drainage de ressources matérielles qui n'est pas négligeable; personne n'a essayé de le calculer.

L'émigration a peut-être eu aussi des avantages pour le Québec. En réduisant la pression démographique et économique, les départs ont pu empêcher l'appauvrissement massif et la baisse du niveau de vie qu'aurait entraînés le surpeuplement. On peut même se demander s'il existe un lien entre, d'une part, un exode aussi important et, d'autre part, les transformations profondes que connaît l'agriculture québécoise à partir de la fin du $\mathrm{xIx}^{\mathrm{e}}$ siècle. Jean H amelin et Yves Roby voient dans la rareté de main-d'œuvre consécutive à l'émigration un facteur qui favorise la mécanisation ${ }^{76}$, mais cette dernière a pu à son tour accélérer les départs. Se pourrait-il aussi que le numéraire apporté des États-Unis par les rapatriés contribue à une meilleure capitalisation des fermes? Cette question n'a jamais été étudiée 7 . Ailleurs dans le monde, il est bien connu que les émigrants contribuent souvent à aider ceux qui sont restés derrière par des transferts d'argent au bénéfice de la parenté. Là encore, à l'échelle nationale, les sommes en cause sont parbis considérables. Qu'en est-il des Canadiens français émigrés? Ont-ils aidé leurs parents du Québec et, si oui, dans quelle mesure? Ces questions mériteraient une étude en profondeur. Au-delà des considérations matérielles, comment le mouvement d'émigration a-t-il marqué la culture au Québec? A-t-il joué un rôle dans l'élargissement du paysage mental et de la perception du monde? A-t-il contribué à une ouverture à la modernité? Le romancier Louis Hémon met dans la bouche de Lorenzo Surprenant une description positive de la ville américaine et de ses avantages ${ }^{78}$. Ces paroles ne suffisent pas à emporter l'adhésion de Maria Chapdelaine, mais si de telles idées circulent au Lac Saint-jean, elles le font aussi ailleurs ${ }^{79}$.

\section{Le phénomène du retour}

Nous n'allons pas tenter de répondre à toutes ces questions ici. Nous voulons surtout examiner un aspect de la rétroaction de cet exode sur le

76. Jean Hamelin et Yves Roby, Histoire économique du Québec, 1851-1896, 201.

77. Elle est soulevée brièvement par Pierre Anctil, «La Franco-Américanie ou le Q uébec d'en bas», Dean R. Louder et Eric Waddell, dir., Du continent perdu.., 33-34.

78. Louis Hémon, M aria Chapdelaine (M ontréal, Fides, 1959), 133-141.

79. À ce sujet, voir Yves Roby, «Émigrés canadiens-français, Franco-Américains de la Nouvelle-Angleterre et images de la société américaine», Gérard Bouchard et Yvan Lamonde, dir. Québécois et Américains, 131-156. 
Québec: I'apport des Franco-Américains - soit nés aux États-Uniş soit y ayant séjourné quelques mois ou quelques années - venus ou revenus s'installer au Québec. L'historien Mark Wyman a montré que les retours en Europe des immigrants aux États-Unis atteignent une ampleur considérable entre 1880 et 1930 et qu'ils ont un impact significatif dans les pays d'origine ${ }^{80}$. Ce phénomène du retour s'est aussi manifesté parmi les Canadiens français, mais il n'a guère été étudié jusqu'ici. Pourquoi ce manque d'intérêt? Faut-il y voir l'effet du syndrome de l'échec du rapatriement officiel qui aurait convaincu les observateurs que les retours n'étaient pas significatifs? S'est-on contenté de chercher, sans les trouver, les rapatriés dans les régions de colonisation, alors que c'était peut-être le dernier endroit où ils auraient souhaité rentrer au pays? Pourquoi ne les a-t-on pas plutôt cherchés dans les vieilles paroisses agricoles ou dans les villes?

Même si peu d'historiens ont étudié spécifiquement le phénomène du retour, plusieurs en ont souligné l'existence dans des travaux sur l'émigration aux États-Unis Nous essaierons de faire ressortir l'apport de cette historiographie tout en poussant un peu plus loin la recherche à propos de certains groupes sociaux.

Voyons d'abord la composition du groupe à l'étude qu'on peut diviser en deux sous-populations, toutes deux d'origine ethnique française:

a) Les expatriés qui, en revenant au Canada, sont considérés comme des rapatriés. Les données du ministère canadien de l'Immigration dans les années 1920 et le recensement de 1931 classent ces rapatriés parmi les immigrants quand ils ont séjoumé trois ans ou plus à l'étranger. Cependant, le recensement de 1941 ramène la limite à un an, ce qui est un peu court ${ }^{81}$. Ces rapatriés sont tous nés au Canada, mais certains ont acquis la citoyenneté américaine, d'autres pas.

b) Les immigrants sans équivoque qui sont nés aux États-Unis IIs appartiennent généralement à la deuxième génération, étant issus de Canadiens qui ont eux-mêmes émigré aux États-Unis. Certains ont une expérience américaine bien courte, puisqu'ils sont encore enfants à leur arrivée, tandis que d'autres immigrent au Canada devenus adultes.

80. Mark W yman, Round-Trip to America. The Immigrants Return to Europe, 1880-1930 (Ithaca, Cornell University Press, 1993), 267 p.

81. Yolande Lavoie, «Les mouvements migratoires... », 80. 
L'ambiguïté entoure également le vocable «Franco-Américain». Pour certains, il ne s'applique qu'aux Canadiens français établis en NouvelleAngleterre, excluant tous ceux qui sont allés ailleurs aux États-Unis. Pour d'autres, il n'a de sens qu'à partir de la deuxième génération. Ces distinctions, peut-être utiles d'un point de vue américain, sont beaucoup moins pertinentes du point de vue québécois

Qui est rapatrié et qui est immigrant? Qui est franco-américain et qui ne l'est pas? À l'évidence, l'usage est flou et, en l'absence de sources précises, il est souvent difficile de départager les uns des autres. Pour les fins de cette étude, la définition la moins restrictive possible a été retenue. Sont donc considérés ici tous les Canadiens français et tous leurs descendants ayant séjourné où que ce soit aux États-Unis ou y étant nés et qui sont ensuite venus vivre au Québec de façon temporaire ou permanente. Les expressions «rapatrié» ou «Franco-Américain» y sont utilisées indistinctement pour les désigner collectivement, à moins d'indication contraire.

Mesurer la taille de ce groupe de migrants s'avère toutefois une tâche ardue. II n'existe aucune statistique fiable avant les années 1920 et les auteurs qui se sont risqués à produire des estimés ont dû le faire à partir de sources partielles et en recourant à des calculs complexes. L'économiste Gilles Paquet a étudié les mouvements migratoires des Canadiens français vers la N ouvelle-Angleterre. Pour la seule décennie 1881-1891, il conclut que 26152 d'entre eux sont revenus au Québec ${ }^{82}$. De son côté, le géographe Ralph D. Vicero, après avoir estimé à 340000 «le nombre net d'immigrants canadiens-français» vers la même région entre 1850 et 1900, ajoute: «ll est probable que le même nombre ait déménagé de façon temporaire ${ }^{83}$. » Par ailleurs, la démographe Yolande Lavoie a mesuré l'émigration de l'ensemble des Canadiens vers les États-Unis entre 1840 et 1940. Si elle ne se risque pas à calculer le nombe de rapatriés pour le $\mathrm{xix}^{\mathrm{e}}$ siècle, elle se sent en terrain plus sûr pour le $\mathrm{xx}^{\mathrm{e}}$. Elle constate que le phénomène du retour s'accentue alors et qu'il devient considérable pendant les années 1920. Au total, pour les quatre premières décennies du siècle, elle estime que près de 500000 Canadiens sont rentrés au pays en provenance des États-Unis; elle ajoute que «la

82. Gilles Paquet, «L'émigration des Canadiens français vers la Nouvelle-Angleterre, 18701910: prises de vue quantitatives», Recherches sociographiques, 5,3 (septembre-décembre 1964): 338-341.

83. Ralph D. Vicero, «Sources statistiques pour l'étude de l'immigration et du peuplement canadien-français en N ouvelle-Angleterre au cours du $\mathrm{xIx}^{\mathrm{e}}$ siècle», Recherches sociographiques, 12,3 (septembre-décembre 1971): 361. 
population francophone de la N ouvelle-Angleterre a pu perdre ainsi, au début du $\mathrm{xx}^{\mathrm{e}}$ siècle, 100000 personnes et peut-être plus encore ${ }^{84} \gg$. II faudrait en outre tenir compte des rapatriés qui sont allés ailleurs qu'en Nouvelle-Angleterre et pour lesquels personne n'a avancé de chiffre. Chose certaine, le recensement de 1941 permet de relever au Québec environ 70000 Canadiens français de retour des États-Unis: les 34336 Américains d'origine française et au moins $80,9 \% 85$ des 43771 natifs du Québec ayant eu les États-Unis comme demier pays de résidence, soit 35413 personnes ${ }^{86}$. Le nombre de francophones revenus ou ayant immigré des États-Unis au Québec entre 1840 et 1940 est donc considérable, même s'il ne touche qu'une minorité des émigrants: il dépasse certainement les 200000 et se rapproche peut-être des 450000 .

II y a lieu de s'interroger sur l'apport de ces rapatriés au Québec, compte tenu de leur expérience américaine. À cet égard, il faut tenir compte de la durée du séjour aux États-Unis Yolande Lavoie constate qu'au $x^{e}$ siècle, les retours sont beaucoup plus susceptibles de toucher ceux dont le séjour est bref (moins de trois ans), que les autres ${ }^{87}$. II faut aussi se rappeler qu'une partie importante des rapatriés sont des enfants revenant avec leur famille; l'expérience américaine ne les a pas touchés autant que les adultes Selon Martine Rodrigue, en 1901, parmi les familles franco-américaines du quartier montréalais de Saint-Jacques dont au moins un des enfants est né aux États-Unis, plus du tiers de ces derniers ont un an ou moins lors de leur retour au Canada et les deux tiers, cinq ans ou moins ${ }^{88}$.

II est inutile de chercher à aller plus loin du côté de la mesure des effectifs. Concentrons-nous plutôt sur d'autres sources, souvent de nature qualitative, pour repérer des Québécois francophones et des Franco-Américains de naissance rentrés au pays après avoir vécu un certain temps aux États-Uniş tout en sachant qu'elles ne révèlent qu'une partie du phénomène.

84. Yolande Lavoie, «Commentaires», Vie française, numéro spécial «Premier colloque de I'Institut français», (1980), 31; voir aussi id., «Les mouvements migratoires des Canadiens... », 79-82.

85. Pourcentage de la population du Québec qui est d'origine française en 1941.

86. Recensement du Canada, 1941, IV, tableaux 20 et 24.

87. Yolande Lavoie, «Les mouvements migratoires des Canadiens... 》, 81.

88. Martine Rodrigue, Les Franco-Américains à Montréal au début du $x x^{e}$ siècle, mémoire de maîtrise (histoire), Université du Québec à Montréal, 1997, 34; ce mémoire a été réalisé sous notre direction; voir aussi Martine Rodrigue, «Les Franco-Américains à M ontréal en 1901: un regard sur le retour au pays», Francophonies d'Amérique, 9 (1999) : 109. 


\section{Les migrations temporaires}

Si l'émigration a longtemps été vue comme une solution de désespoir ou de dernier recours, en réaction aux difficultés économiques prévalant dans certaines régions du Québec, Bruno Ramirez, s'appuyant sur l'étude comparée des migrations internationales, invite à la concevoir comme une stratégie rationnelle. En effet, les individus et les familles de l'époque ont un éventail de possibilités: s'adonner à la colonisation dans les régions en développement, au Québec ou même dans I'Ouest canadien, aller vers les villes industrielles du Québec ou vers celles de la Nouvelle-Angleterre ou encore tenter l'aventure du Midwest ou de l'Ouest américain. Le choix de l'une ou l'autre ou de plusieurs de ces stratégies relève le plus souvent de la famille et s'appuie sur les réseaux de parenté et de voisinage. II dépend des perspectives et des aspirations individuelles et familiales, de la conjoncture économique, de l'âge des personnes, de leur état civil, de la taille et de la composition des familles, de la présence de connaissances ou de parents au lieu d'arrivée. Ces choix s'inscrivent dans un contexte où la mobilité des individus et la migration, de courte ou de longue distance, font partie de l'expérience commune des Québécois pendant la seconde moitié du xix ${ }^{\mathrm{e}}$ et les premières décennies du $x^{\mathrm{e}}$ siècle ${ }^{89}$. Frances Early illustre aussi, à partir de l'exemple de Félix Albert, le caractère rationnel des choix de l'émigrant qui, selon elle, se rattachent à une éthique du travail de type capitaliste ${ }^{0}$.

Parmi les émigrants québécois de l'époque, certains veulent rompre définitivement avec la misère, d'autres cherchent simplement à se refaire une santé financière dans le but de reprendre sur un pied plus solide leur activité antérieure, d'autres encore veulent acquérir une expérience nouvelle. Nombreux sont ceux qui ne conçoivent leur départ que comme une solution temporaire. La majorité des émigrants viennent du monde rural. Un grand nombre ont connu les frustrations et les misères

89. Bruno Ramirez, Par monts et par vaux..., 36-53, 85-105, 159-173; Yves Roby, «Partir pour les États», Serge Courville, dir., Atlas historique du Québec. Population et territoire (Québec, Les Presses de l'Université Laval, 1996), 121-131; Yves Frenette, «Macroscopie et microscopie d'un mouvement migratoire: les Canadiens français à Lewiston au xix ${ }^{e}$ siècle», Yves Landry et al., dir., Les chemins de la migration en Belgique et au Québec du xvII au xxesiècle (Beauport, MNH, 1993), 221232.

90. Frances H. Early, «The Rise and Fall of Felix Albert: Some Reflections on the Aspirations of Habitant Immigrants to Lowell, M assachusetts in the Late Nineteenth Century », Raymond Breton et Pierre Savard, dir., The Quebec and Acadian Diaspora in North America (Toronto, Multicultural History Society of Ontario, 1982), 25-38. 
de la vie de colons, plusieurs appartiennent au fort groupe des journaliers qu'engendre le surpeuplement agricole, tandis que d'autres sont de petits cultivateurs dont l'exploitation n'est pas suffisamment importante pour soutenir une famille. La plupart n'arrivent pas à vivre de l'agriculture et doivent recourir à des activités complémentaires en travaillant comme engagés, bûcherons, etc. Un phénomène moins connu est celui de l'émigration des urbains. Par exemple, les maigres salaires payés par l'industrie textile du Québec, en comparaison avec ceux qu'on peut obtenir en N ouvelle-Angleterre, peuvent-ils constituer une incitation au départ?

Pourtant, de nombreux Q uébécois ayant vécu l'expérience américaine choisissent ensuite de rentrer. Comment expliquer ces retours? Les entrevues réalisées par Jacques Rouillard avec d'anciens rapatriés permettent de saisir quelques-unes des raisons: I'atteinte de l'objectif de se refaire une santé financière en vue de reprendre l'exploitation agricole, le mal du pays, la préférence pour l'agriculture et l'inadaptation au travail industriel. Yves Roby insiste sur les aléas de la conjoncture, les crises économiques qui sévissent périodiquement aux États-Unis eprésentant des temps forts de retour. D'autres mettent en lumière des facteurs structurels. Pour Bruno Ramirez, l'arrivée des enfants, encore trop jeunes pour aller travailler, incite plusieurs couples à rentrer; ce phénomène est renforcé, au début du $\mathrm{xx}^{\mathrm{e}}$ siècle, par les nouvelles lois américaines bannissant le travail des enfants en manufacture. En fait, le projet migratoire n'est rentable pour les familles que si elles ont des adolescents et de jeunes adultes en âge de travailler. Jacques Rouillard évoque, surtout pour les années 1920, le déclin inexorable de l'emploi dans l'industrie textile de la Nouvelle-Angleterre ${ }^{91}$.

La migration a souvent les allures d'un va-et-vient entre le Québec et la Nouvelle-Angleterre. Après un séjour aux États-Unis, certains migrants reviennent à leur point de départ et se remettent à l'agriculture, mais s'ils échouent, ils reprennent la route du sud et ces tentatives peuvent se répéter à quelques reprises au cours d'une vie. Pierre Anctil fournit des exemples de ces mouvements pendulaires. Bruno Ramirez constate que, dans le comté de Berthier, les deux tiers des

91. Jacques Rouillard, Ah les États! Les travailleurs canadiens-français dans l'industrie textile de la Nouvelle-Angleterre d'après les témoignages des derniers migrants (Montréal, Boréal Express, 1985), 77-82; Yves Roby, Les Franco-Américains..., 54-57; Bruno Ramirez, Par monts et par vaux..., 46-48, 139-144, 146-148. 
familles rapatriées qu'il a relevées dans les listes nominatives du recensement de 1881 ne sont plus là dix ans plus tard, ayant à nouveau migré ailleurs, sans qu'on sache si c'est au Québec ou aux États-Unis Les listes nominatives, qui indiquent le pays de naissance de chaque personne, permettent d'observer les familles qui participent à de tels allers-retours. Ainsi, dans le comté de Saint-Jacques en 1901, Martine Rodrigue a repéré une famille ayant fait au moins trois séjours aux États-Unis, entrecoupés de retours au Québec, chaque phase étant marquée de naissances de l'un ou l'autre côté de la frontière ${ }^{92}$.

Si les retours provisoires peuvent apparaître comme un échec, ce n'est pas nécessairement le cas pour les retours permanents, après un ou des séjours temporaires d'une durée plus ou moins longue aux États-Unis Les migrants reviennent avec des épargnes qui sont injectées dans l'économie québécoise. Ils ont souvent appris de nouveaux métiers, acquis des expériences, des habiletés ou des connaissances techniques qu'ils peuvent mettre à profit. La migration de retour devient ainsi, pour le Québec, synonyme d'apport de capital monétaire et de capital d'information.

\section{Le mirage du rapatriement dirigé}

Dans les dernières décennies du xix ${ }^{\mathrm{e}}$ siècle, interpellées par l'ampleur de l'émigration, les élites politiques et cléricales du Québec élaborent un discours sur le rapatriement. Ce discours est presque exclusivement centré sur la colonisation, perçue comme la seule façon de réintégrer les expatriés, et sur la sélection des candidats les plus aptes à réussir dans ce type d'activité. II conduit le gouvernement québécois à financer les activités d'agents de recrutement chargés d'inciter et d'aider les FrancoAméricains à se faire colons $9^{33}$.

La mesure la plus importante vient en 1875 avec une loi visant à encourager la colonisation, notamment par des rapatriés. Elle prévoit l'octroi de conditions particulières aux colons et le versement d'une

92. Pierre Anctil, Aspects of Class Ideology in a New England Ethnic Minority: The FrancoAmericans of Woonsocket, Rhode Island (1865-1929), thèse de PhD, New York, New School for Social Research, 1980, 46-54; Bruno Ramirez, Par monts et par vaux... , 46-48; Martine Rodrigue, «Les Franco-Américains... », 112.

93. Ce discours est analysé en détail par Martin Pâquet, "Le meilleur immigrant": le rapatrié des États-Unis comme catégorie pour les responsables politiques du Canada-Uni et du Québec, 1849-1968», Francophonies d'Amérique, 9 (1999) : 87-105; voir aussi Yves Roby, «Les Canadiens français des États-Unis (1860-1900): dévoyés ou missionnaires», RHAF, 41,1 (été 1987) : 3-22. 
avance en argent. Les efforts sont concentrés sur trois cantons du district de Saint-François et surtout à La Patrie. La crise économique qui sévit à l'époque et qui réduit l'emploi dans les manufactures incite un certain nombre de Franco-Américains à s'installer dans la nouvelle colonie. Les conditions d'établissement sont difficiles et plusieurs terres ne sont guère propices à l'agriculture. Au bout du compte, le projet s'avère un échec. Selon J. I. Little, seulement quelques centaines de colons sont des apatriés et la plupart repartent quand la situation économique s'améliore aux États-Unis, de sorte que la région est surtout habitée par des ruraux venus d'autres parties du Québec. Dès 1877, le gouvernement québécois met fin à l'expérience ${ }^{94}$. Ferdinand Gagnon, le joumaliste franco-américain qui avait été embauché par le Québec comme agent de ecrutement, en sort désabusé; il cessera par la suite de prêcher le retour au pays et se fera l'apôtre de la naturalisation des Franco-Américains ${ }^{95}$.

Les Franco-Américains sont aussi dans la mire des sociétés de colonisation qui naissent dans diverses régions du Québec ${ }^{96}$. L'une d'elles, la Société de rapatriement et de colonisation du Lac Saint-jean, réussit à en attirer plusieurs centaines entre 1896 et 1904, avec I'appui financier du gouvernement fédéral. Mais, selon Robert G. LeBlanc, la plupart d'entre eux abandonnent rapidement. La dureté des conditions dans cette zone encore éloignée décourage plusieurs de ceux qui s'étaient déjà acclimatés au milieu urbain de la N ouvelle-Angleterre ${ }^{97}$. La colonisation redeviendra à la mode beaucoup plus tard, quand la crise des années 1930 entraînera la fermeture de filatures importantes; des Franco-Américains opteront alors pour cette solution de demier recours contre la misère urbaine, mais on ne connaît pas les effectifs en cause $e^{98}$.

La politique officielle de rapatriement dirigé vers les régions de colonisation, mise en œuvre au $\mathrm{xIx}^{\mathrm{e}}$ siècle, s'avère donc un échec, constaté par tous les historiens qui ont abordé la question. Ce qu'on oublie trop souvent de dire, c'est que cette politique n'a touché qu'une infime partie

94. J. I. Little, Nationalism, Capitalism and Colonization in Nineteenth-Century Quebec: The Upper St. Francis District (M ontréal, M cGill-Q ueen's University Press, 1989), 158-173.

95. Donald Chaput, «Some Repatriement Dilemmas», Canadian Historical Review, 49,4 (décembre 1968) : 402-406; Yves Roby, «Ferdinand Gagnon», DBC, XI : 362-364.

96. Nous ne traiterons pas ici des efforts du gouvernement fédéral et des dirigeants francomanitobains pour orienter des Franco-Américains vers la colonisation dans l'O uest canadien.

97. Robert G. LeBlanc, «Colonisation et rapatriement au Lac Saint-Jean (1895-1905) », RHAF, 38,3 (hiver 1985): 379-408; voir aussi id., «Regional Competition for Franco-American Repatriates, 1870-1930», Q uébec Studies, 1,1 (printemps 1983): 110-129.

98. Jacques Rouillard, Ah les États!..., 81. 
de ceux qui sont revenus des États-Unis Pour plusieurs, repartir à zéro en défrichant une terre boisée dans une région pionnière n'est pas une solution de rechange à la vie, même difficile, dans une ville de la Nouvelle-Angleterre. Le mouvement de retour au Québec ne se fait pas principalement à l'enseigne du rapatriement dirigé, mais à celle du rapatriement spontané, sans encadrement gouvernemental ou autre. II est le résultat de décisions individuelles s'inscrivant logiquement dans les stratégies migratoires évoquées précédemment. Les informations dont nous disposons à propos des individus qui reviennent au Québec indiquent deux tendances D'une part, plusieurs d'entre eux cherchent d'abord à revenir dans leur paroisse de départ et à reprendre les choses là où ils les avaient laissées. D'autre part, de nombreux rapatriés, forts d'une expérience urbaine et industrielle récemment acquise, se dirigent vers les villes du Québec. Dans ce dernier cas, le passage par les ÉtatsUnis n'aura été qu'une étape dans le processus d'exode rural, de migration de la campagne vers la ville.

\section{Des élites aguerries}

En 1894, le journaliste Télesphore Saint-Pierre, ayant lui-même vécu longtemps au Michigan, publie son H istoire du commerce canadien-français de M ontréal. L'ouvrage contient un répertoire de biographies d'hommes d'affaires et de représentants des professions libérales, la plupart membres de la Chambre de commerce. On y trouve 98 notices concernant 111 individus (certaines traitent de quelques membres d'une même famille). Sont-elles représentatives de la bourgeoisie francophone de Montréal ? Il est difficile de le dire, mais la présence dans cette galerie de portraits d'hommes d'affaires importants et d'entrepreneurs plus modestes donne à penser qu'il s'agit d'un échantillon intéressant. Or 17 de ces notices, traitant de 19 individus (soit 17\% du total), concernent des hommes qui ont vécu un certain temps aux États-Unis Sans doute Saint-Pierre est-il personnellement sensible à cette dimension de la carrière de ses sujets et n'hésite-t-il pas à la signaler. L'existence de ces notices biographiques permet de tracer un portrait collectif de ce groupe de Franco-Américains ${ }^{9}$.

Un seul d'entre eux est né aux États-Uniş le fils d'un patriote fuyant la répression consécutive aux Rébellions. Tous les autres sont du

99. Télesphore Saint-Pierre, Histoire du commerce canadien-français de M ontréal, 1535-1993. Un souvenir (M ontréal, Sabiston Litho \& Publishing, [1894]; réédition: Montréal, Élysée/ Commerce, 1975), $136 \mathrm{p}$; l les notices biographiques sont aux pages 101-135. 
Québec: 2 sont nés à M ontréal, 1 à Québec et 14 dans le monde rural, sans que l'on sache si c'est en milieu villageois ou sur une feme (le lieu de naissance d'un des sujets est inconnu). A l'exception des frères M orin, de Gentilly, les ruraux proviennent de la région de Montréal et leur stratégie migratoire est intéressante: 11 quittent leur milieu d'origine pour aller aux États-Uniş avant d'arriver à Montréal; seulement 2 vont d'abord à Montréal, puis aux États-Unis arant de revenir dans la métropole. Ce constat semble confirmer le rôle transitoire qu'a parfois l'expérience américaine dans le processus d'exode rural.

La durée du séjour américain est connue dans la majorité des cas: 4 y vivent plus de 10 ans et 6 de 2 à 6 ans tandis qu'on relève aussi les mentions «plusieurs années» (1, probalement de 8 à 9 ans), «quelques années» (1, probablement de 5 à 7 ans), «quelque temps» (5) et «quelques mois» (1); quant à celui pour lequel aucune durée rest indiquée, elle est vraisemblablement de plusieurs années puisqu'il n'arrive à Montréal qu'à l'âge de 29 ans Les destinations américaines sont très variées: deux choisissent l'État de $\mathrm{N}$ ew York, tandis que le Rhode Island, le Massachusetts, le Michigan, I'Illinois, le Wisconsin, le Minnesota et la Californie en accueillent un chacun; un va à la fois à Chicago et en Arkansas, trois dans l'est des États-Uniş un dans le nord, un autre parcourt plusieurs villes, de N ew York à la N ouvelle-Orléans, et pour les quatre derniers, la destination n'est pas précisée.

Le texte des notices permet de constater que, dans plusieurs cas, le passage aux États-Unis fournit une occasion d'apprentissage ou de perfectionnement. Ainsi, le séjour du phamacien Séraphin Lachance lui permet «d'acquérir une plus grande somme d'expérience, tant pharmaceutique que commerciale»; Joseph Asselin en profite pour suivre un cours commercial tout en «s'initiant à la vie des affaires»; Pierre Dansereau, formé à M ontréal, prend de l'expérience auprès d'un carossier du Rhode Island; Albert J. Corriveau apprend à $\mathrm{New}$ York la fabrication de la soie et se lance dans cette production à Montréal; le peintredécorateur D.-A. Beaulieu «se perfectionna dans son art»; c'est aussi le cas pour plusieurs autres. De son côté, Alphonse Pigeon tient un magasin général en Illinois pendant 17 ans ${ }^{100}$. Plusieurs sont en mesure de se lancer en affaires dès leur arrivée à M ontréal, grâce aux économies accumulées là-bas; les autres le font après avoir travaillé quelques années comme employés. Quand on examine l'éventail des professions exercées 
à Montréal vers 1894 par ces migrants, on relève 7 commerçants, 4 industriels, 3 entrepreneurs liés à la construction, 1 financier, 2 pharmaciens, 1 médecin et 1 ingénieur.

D'autres répertoires biographiques contiennent des notices sur plusieurs hommes d'affaires de Montréal et d'ailleurs au Québec ayant passé une partie de leur vie aux États-Unis pendant la période qui va, en gros, de 1840 à 1914. Arrêtons-nous à quelques exemples. Chez les industriels, Augustin Cantin, fondateur d'un important chantier naval à Montréal, prend d'abord de l'expérience en travaillant dans des établissements de Liverpool et de N ew York ${ }^{101}$. Les premiers manufacturiers de chaussures canadiens-français - Guillaume Bresse, Guillaume Boivin et Louis Côté - vivent un certain temps au Massachusetts où ils sont initiés aux nouvelles méthodes de la production en série ${ }^{202}$. Pamphile Biron, qui est parti à l'âge de 7 ans rentre au Québec 12 ans plus tard et fabrique des voitures à Sherbrooke ${ }^{103}$. Joseph $\mathrm{E}$. D upré, né à Worcester en 1876, vient faire une partie de ses études au Québec, puis s'installe en affaires à Brockton, au Massachusetts, où il est même élu conseiller municipal ; en 1904, il déménage dans la métropole et y monte une usine de formes de chaussures ${ }^{104}$.

Des marchands tels Pierre-Paul Martin à Montréal, Abraham Lomme à Saint-Jean et quelques autres séjournent aux États-Unis pour y faire des études ou l'apprentissage du commerce ${ }^{105}$. Certains hôteliers acquièrent une solide expérience dans la république voisine, tels Joseph Riendeau qui y vit 17 ans ou Paul C. Lévesque qui passe 15 ans en Californie ${ }^{106}$.

Du côté des professions libérales, il est très rare de trouver des avocats ou des notaires qui traversent la frontière, probablement à cause de la spécificité du droit civil québécois et de la nature de la formation. II y a tout de même l'avocat Daniel Bergevin qui, après dix ans de pratique à Montréal, s'en va à Chicago où il réside neuf ans et joue un rôle actif en politique avant de rentrer dans la métropole et de s'y lancer en affaires ${ }^{107}$.

101. W m Cochrane, The Canadian Album. Men of Canada or Success by Example (Brantford, Bradley Garetson, 1895), IV : 512; voir aussi Gerald J. J. Tulchinsky, The River Barons..., 209.

102. Jean Benoit, «Guillaume Bresse», DBC, XII: 137-138; Joanne Burgess, «Guillaume Boivin», DBC, XIV : 103-106; Peter Gossage, «Louis Côté», DBC, XIV : 262-263.

103. J. Castell Hopkins, The Canadian Album, V : 301.

104. Lorenzo Prince, M ontreal, Old and N ew (M ontréal, International Press Syndicate, 1915), 394.

105. Voir, par exemple, W m Cochrane, The Canadian Album, II : 241, 292, 468; IV : 47, 243, 261; Lorenzo Prince, M ontreal, Old and N ew, 348, 431.

106. Wm Cochrane, The Canadian Album, IV : 213, 298, 379, 427.

107. Lorenzo Prince, Montreal, Old and N ew, 498. 
II y a aussi le notaire Eugène Archambault, de Saint-Jean, qui passe deux ans en Californie ${ }^{108}$. La situation est différente chez les médecins. Quelques-uns vont pratiquer là-bas un certain nombre d'années avant de rentrer au Québec ${ }^{109}$. D'autres choisissent d'étudier ou de se spécialiser aux États-Unis ${ }^{110}$, à une époque où la plupart des médecins québécois qui vont à l'étranger se dirigent vers Paris; ils préfigurent une réorientation qui gagnera en importance au cours du $\mathrm{xx}^{\mathrm{e}}$ siècle. Des dentistes font de même à Philadelphie ${ }^{111}$. Des ingénieurs vont travailler aux États-Unis où ils acquièrent une précieuse expérience ${ }^{112}$.

Le clergé participe aussi à ce mouvement. Des milliers de prêtres, de religieux et de religieuses du Québec œuvrent dans des établissements franco-américains. Pour plusieurs, ce sejour n'est qu'une étape dans une carrière qui se déroule des deux côtés de la frontière. Quelles traces laisse-t-il dans leur façon de pratiquer leur ministère, une fois de retour au Québec?

La vie franco-américaine compte pour beaucoup dans la frmation de quelques journalistes québécois dont certains atteignent la célébrité. Au premier rang vient Honoré Beaugrand, l'un des chefs de file du libéralisme radical au Québec. II passe plusieurs années dans la république américaine, surtout à Fall River, où il fonde deux journaux, et devient un porte-parole des Franco-Américains II s'établit à Montréal en 1878 et, l'année suivante, fonde La Patrie, bientôt l'un des plus importants quotidiens de la métropole. Beaugrand s'enrichit à Montréal et il est aussi maire de la Ville de 1885 à 188713. Frédéric H oude émigre en 1869 et collabore à quelques journaux franco-américains. Rentré à M ontréal en 1875 , il se joint à la rédaction de l'organe ultramontain Le N ouveau M onde, dont il sera propriétaire de 1879 à 1882. II siège en outre comme député conservateur fédéral de 1878 jusqu'à sa mort, en $1884^{114}$.

II y a aussi Olivar Asselin, auquel Hélène Pelletier-Baillargeon a consacré une imposante biographie. Asselin émigre avec sa famille en 1892, à l'âge de 17 ans, et connaît pendant un an et demi le dur labeur des

108. J. Castell Hopkins, The Canadian Album, V : 159.

109. Wm Cochrane, The Canadian Album, II : 177, 184, 307; IV : 284, 384; J. Castell Hopkins, The Canadian Album, V : 106.

110. W m Cochrane, The Canadian Album, II : 159; IV : 303; Lorenzo Prince, M ontreal, Old and New, 456, 457, 462.

111. W m Cochrane, The Canadian Album, IV : 126, 370.

112. Ibid., II : 453; III : 39; Lorenzo Prince, Montreal, Old and New, 238, 472.

113. François Ricard, «H onoré Beaugrand», DBC, XIII : 56-58.

114. David M. Hayne, «Frédéric Houde», DBC, XI : 467-468. 
filatures. II amorce ensuite une carrière de journaliste au service de diverses publications. En 1898, lors de la guerre contre l'Espagne, il s'enrôle dans l'armée américaine, mais la maladie l'éloigne du champ de bataille. C'est en 1900 qu'il s'installe à Montréal et se lance dans le journalisme de combat, se distinguant en particulier à la direction du Nationaliste. Sa période franco-américaine ne dure que huit ans, mais elle le marque de façon importante. C'est là que se forment ses idées à propos de la lutte nationaliste, du sort des ouvriers, de la faiblesse des politiciens ${ }^{115}$. D'autres journalistes - Ludger Duvernay, Médéric Lanctot, Louis Fréchette, Rémi Tremblay, Thomas Côté, Télesphore Saint-Pierre, Alfred Duclos Decelles et Jean-Léon Kemmer-Laflamme ont à leur actif un séjour américainn ${ }^{16}$.

Le monde des arts n'échappe pas à l'attraction américaine. Des répertoires biographiques soulignent le passage aux États-Unis de photoraphes et de musiciens ${ }^{117}$. David Karel a identifié un grand nombre de peintres et de sculpteurs québécois qui œuvrent au sud de la frontière ou y acquièrent une partie de leur formation, dont Ozias Leduc et MarcAurèle Fortin ${ }^{118}$. Au théâtre, le plus important comédien et dramaturge québécois des premières décennies du $\mathrm{xx}^{\mathrm{e}}$ siècle, Julien Daoust, fait deux longs séjours aux États-Unis entre 1888 et 1898 et, en plus d'y jouer, il est en mesure «de découvrir les progrès techniques de la scène newyorkaise» qu'il appliquera ensuite à M ontréa|119. Le milieu des arts représente toutefois un champ particulier dans la mesure où les artistes sont aussi très attirés par l'Europe, surtout par Paris, mais il faut souligner l'importance que prennent les États-Unis dans leur formation et leur carrière, dès le $\mathrm{xIx}^{\mathrm{e}}$ siècle.

Du côté de l'élite syndicale, on peut se demander quel a été le rôle des Franco-Américains dans la diffusion du syndicalisme intemational au

115. Hélène Pelletier-Baillargeon, O livar Asselin et son temps Le militant (Saint-Laurent, Fides, 1996), $780 \mathrm{p}$.

116. Jean-M arie Lebel, «Ludger Duvernay», DBC, VIII : 286-292; Jean Hamelin, «M édéric Lanctot», DBC, X: 461-467; Jacques Blais, «Louis Fréchette», DBC, XIII: 388-392; Wm Cochrane, The Canadian Album, II: 109; H.J. Morgan, Canadian Men and Women of the Time (Toronto, 1912), 262; Yves Roby, «T élesphore Saint-Pierre», DBC, XIV : 985-986; J. Castell Hopkins, The Canadian Album, V: 19.

117. W m Cochrane, The Canadian Album, IV : 378, 486; J. Castell Hopkins, The Canadian Album, V : 113, 157, 171, 261, 312, 314.

118. David Karel, "L'expérience continentale de l'art québécois au $\mathrm{xIx}^{\mathrm{e}}$ siècle», Gérard Bouchard et Yvan Lamonde, dir., Q uébécois et Américains..., 197-225.

119. Jean-Cléo Godin, «ulien Daoust dramaturge, 1866-1943», Theatre History in Canada/ Histoire du théâtre au Canada, 4,2 (automne 1983): 115. 
Québec. Le plombier Alphonse Verville, après plus d'une dizaine d'années de travail à Chicago, s'établit à M ontréal et occupe divers postes à la direction du mouvement ouvrier, jusqu'à la présidence du Congrès des métiers et du travail du Canada; il est aussi élu député ouvrier à la Chambre des Communes ${ }^{120}$. S'agit-il d'un cas isolé?

II faudrait dépouiller bien d'autres sources pour arriver à dresser la cartographie, tant sociale que spatiale, des élites franco-américaines au Québec. Ces exemples tirés de divers répertoires biographiques permettent tout de même quelques constatations Pour la période 1840-1914, les carrières de ces membres des élites, locales ou nationales illustrent un phénomène d'ascension sociale (la nature des sources contribue évidemment à cette perception). Dans la plupart des cas, cette mobilité sociale passe par une mobilité géographique, à la fois de la campagne vers la ville et à travers l'espace nord-américain. On remarque d'ailleurs que l'itinéraire de plusieurs de ces personnes n'est pas limité à la N ouvelle-Angleterre et qu'il passe souvent par le M idw est et même par la Californie. Dans la plupart des cas, le séjour américain apparaît comme une étape de la carrière, qui permet l'apprentissage ou l'approfondissement tout autant de la langue angaise que de la maîtrise d'un métier ou d'une profession, de ses méthodes et de ses techniques

\section{Une population insaisissable?}

Les répertoires biographiques sont toutefois d'un bien piètre secours pour connaitre la situation de la masse des Franco-Américains, ces travailleurs ou ces agriculteurs qui par milliers reviennent au Québec. Les listes nominatives du recensement permettent d'en retracer un certain nombre. La présence, dans une famille, d'un ou de plusieurs enfants nés aux États-Unis y constitue le marqueur de repérage. En l'absence d'un tel événement démographique, il est impossible d'identifier un émigrant rentré au pays.

Bruno Ramirez a utilisé cette source pour le comté de Berthier, de 1871 à 1891. Ses observations concernent surtout la structure démographique des ménages, ce qui lui permet d'associer le retour à la présence d'enfants en bas âge. Toutefois, pour deux paroisses en 1881, il indique la profession du chef de ménage chez les rapatriés: dans $40 \%$ des cas où I'information est précisée, c'est un cultivateur et, dans la même proportion, un journalier; s'y ajoutent quelques artisans, des voyageurs, un 
médecin et une veuve. On ne sait pas si cette répartition ressemble ou non à celle qui prévaut dans la population de ces paroisses ${ }^{121}$.

Pour l'étude des Franco-Américains du quartier Saint-Jacques, à Montréal, Martine Rodrigue utilise les listes nominatives du recensement de 1901 qui présentent des informations plus riches que celles des recensements antérieurs. On y trouve notamment l'année d'immigration au Canada et une indication claire de la relation entre chaque résidant et le chef de ménage. L'auteure est ainsi en mesure d'identifier deux groupes. Le premier est formé de 357 familles canadiennesfrançaises dont au moins un membre est né aux États-Unis, pour un total de 1705 personnes dont 512 sont américaines de naissance. Parmi ces familles, 166 ont un ou, rarement, les deux conjoints qui sont américains, mais le recensement ne permet pas de savoir si l'ensemble de la famille ou seulement le conjoint a vécu aux États-Unis Les 191 autres familles ont au moins un enfant né aux États-Uniş ce qui confirme un séjour familial au sud de la frontière. Parmi ces dernières, $52 \%$ sont rentrées au pays au cours de la décennie précédente, 30\% dans les années 1880, 18\% plus de vingt ans auparavant et la majorité des retours surviennent quand tous les enfants sont âgés de 10 ans ou moins En outre, le quart d'entre elles sont, au moment du recensement, dirigées par un veuf ou le plus souvent une veuve, ce qui semble indiquer que le veuvage puisse être, surtout pour les femmes, un motif de retour ${ }^{122}$.

Parmi l'ensemble des 357 familles, 36\% de leurs membres qui ont un emploi sont dans la fabrication, principalement dans la confection, la chaussure et l'imprimerie; $20 \%$ œuvrent dans les services de toute sorte, $16 \%$ dans le commerce, près de $6 \%$ dans les transports et $5 \%$ sont journaliers. Près de la moitié de cette main-d'œuvre est formée d'ouvriers, et $12 \%$ sont des gens d'affaires, dont plusieurs commerçants. Ces données indiquent donc une assez grande diversité qui peut s'expliquer par la nature de l'économie montréalaise. II n'est cependant pas possible de connaître leur représentativité puisque aucun tableau des professions n'a été compilé pour le recensement imprimé de 1901, malgré l'information recueillie dans les questionnaires. La nature du recensement ne permet pas non plus d'établir un lien entre l'emploi occupé à Montréal et une quelconque expérience de travail aux ÉtatsUnis, sinon de dire que, compte tenu de leur âge au moment de leur

121. Bruno Ramirez, Par monts et par vaux..., 45-48.

122. M arine Rodrigue, Les Franco-Américains..., 24-44. 
retour, une nette majorité des chefs de famille avaient certainement acquis une certaine expérience ${ }^{123}$.

Martine Rodrigue identifie par ailleurs un deuxième groupe de 126 célibataires, nés aux États-Unis et d'origine française, qui ne vivent pas avec leur famille immédiate. Ils forment un groupe disparate comprenant en majorité des femmes et beaucoup d'enfants. Près de la moitié (57) habitent seuls ou en pension, tandis que 17 enfants vivent dans une famille apparentée (grands-parents, etc.). Les autres se retrouvent dans des institutions: 14 sont membres d'une communauté religieuse, 17 sont pensionnaires au couvent de la $\mathrm{M}$ iséricorde qui accueille les mères célibataires pendant leur grossesse et 21 sont à l'École de réforme, dans un orphelinat ou dans une école de sourdes et muettes. Ces derniers cas semblent indiquer que des familles franco-américaines ont recours aux établissements spécialisés de Montréal pour y placer leurs enfants qui présentent des problèmes particuliers ${ }^{124}$.

Le mémoire de Martine Rodrigue constitue pour l'instant la seule étude approfondie de l'ensemble des rapatriés franco-américains vivant dans une partie du Québec et identifiables dans les listes nominatives des recensements. Celles-ci livrent des résultats intéressants, mais limités. Pour les exploiter pleinement, il faudrait pouvoir les jumeler arec ceux provenant d'autres sources (par exemple, les registres d'état civil ou les rôles d'évaluation). Rappelons cependant qu'une partie des rapatriés échappe à l'œil des recenseurs de cette époque: tous ceux, célibataires ou mariés, qui sont nés au Québec et sont revenus des États-Unis sans y avoir eu d'enfants.

Par ailleurs, les témoignages de migrants ou de personnes qui les ont connus peuvent éclairer notre perception. Ainsi, Gérard Filion raconte dans ses Mémoires les pérégrinations de son père, «né à Rimouski en 1860, élevé à Matane, devenu bûcheron par nécessité au $\mathrm{NeN}$ H ampshire, cabaretier au M assachusetts», qui épouse à Salem, en 1884, sa mère, venue de I'Isle-Verte travailler dans cette ville à l'âge de 18 ans Deux ans plus tard, son père a accumulé suffisamment d'argent pour rentrer au pays et racheter la terre de son beau-père. Filion ajoute que la famille «fera un deuxième séjour aux États à Nashua, de 1896 à 1903», mais que, «sous la pression des enfants qui refusent de vivre en ville et 
de travailler dans les "facteries" », elle revient à l'Isle-Verte ${ }^{125}$. Ce témoignage indique que le séjour américain peut être rémunérateur et permettre un nouvel établissement au Québec. Trouve-t-on beaucoup de cas de ce genre dans le monde rural ? Pour répondre à la question, il faudrait éplucher les témoignages familiaux et les histoires de vie recueillies en diverses parties du Québec ${ }^{126}$.

Jacques Rouillard a réalisé des entrevues avec une cinquantaine de personnes, toutes originaires du monde rural, qui ont travaillé dans les filatures de la Nouvelle-Angleterre, principalement entre 1915 et 1930. La plupart sont revenues au Québec dans les années 1930 ou aant. L'analyse de l'auteur et les extraits d'entrevues qu'il publie révèlent cependant peu de choses sur leur situation zprès ce rapatriement, car ce n'est pas l'objet de l'ouvrage. II semble bien que dans plusieurs cas, le retour s'est fait à la campagne, puisque l'objectif familial était précisément de recueillir aux États-Unis suffisamment d'argent pour régler des dettes ou acheter une nouvelle terre. Certaines personnes se sont tout de même installées en milieu urbain, à M ontréal, Trois-Rivières ou Louiseville. II ressort aussi de certains témoignages que l'arrivée à la campagne ou dans une petite ville pose certains problèmes d'ajustement, surtout pour les jeunes déjà marqués par la culture urbaine américaine ${ }^{127}$. Dans un autre registre, il serait intéressant d'étudier l'image du retour au Québec fournie par la production littéraire.

II est difficile d'aller plus loin dans l'état actuel des recherches. Les travaux disponibles à propos des immigrants franco-américains et des rapatriés canadiens-français installés au Québec ne révèlent que la pointe de l'iceberg. II reste encore beaucoup à faire pour comprendre le phénomène du retour, pour en cerner les rythmes et les modalités, pour dégager une typologie des patterns de réinsertion des migrants et pour en évaluer l'impact sur la société québécoise.

conclusion

Cet état de la question à propos des migrants américains et francoaméricains au Québec permet de dégager un certain nombre d'observa-

125. Gérard Filion, Fais ce que peux. En guise de mémoires (M ontréal, Boréal, 1989), 19, 23-26. Notons que la pression des enfants peut aussi jouer en sens inverse et amener des familles à rester aux États-Unis.

126. Ainsi, Gérard Bouchard nous a signalé que les «mémoires d'anciens» recueillis par la Société historique du Saguenay contiennent plusieurs témoignages en ce sens.

127. Jacques Rouillard, Ah les États!..., passim. 
tions. II faut d'abord souligner l'ancienneté et la durée de ce double mouvement migratoire. Pour les Américains, nous avons proposé une périodisation, mettant successivement en vedette trois figures emblématiques de l'immigrant: celle du colon, celle de l'entrepreneur, celle du gestionnaire et de l'expert. L'état plus fragmentaire de la recherche sur les Franco-Américains rentrés au Québec n'a pas permis d'établir un découpage aussi net, mais notre enquête dans les répertoires biographiques montre que le phénomène a touché les élites dans plusieurs champs d'activité.

Jusqu'ici, ce double mouvement migratoire n'a guère trouvé sa place dans les synthèses générales. Sa prise en compte permettrait pourtant d'éclairer sous un jour nouveau certains aspects de I'histoire du Québec. Ainsi en est-il du débat sur l'américanité. Notre examen de I'historiographie indique que les migrants américains et franco-américains ont été d'importants vecteurs d'américanisation au Québec. Sans être les seuls, ils ont néanmoins joué un rôle fondamental dans le transfert de capital, de savoir-faire, de méthodes de travail, de techniques et de technologie, de modèles architecturaux ou artistiques, d'idées, de manières de vivre et, plus globalement, de culture. Leur trajectoire spatiale, sociale et culturelle en fait également d'importants témoins et vecteurs de l'américanité du Québec. II ne faut cependant pas écarter la possibilité que certains d'entre eux - des prêtres ou des journalistes, par exemple aient au contraire rejeté le modèle américain et valorisé la différence québécoise.

Sur un autre plan, I'histoire de la migration en provenance des ÉtatsUnis montre que les relations américano-québécoises sont loin de se limiter à l'influence du «capital américain», perçu comme expression d'un rapport impérialiste. Bien avant l'arrivée des grandes entreprises et de leurs filiales, il y a plus d'un siècle de présence d'immigants venus avec un modeste capital, souvent beaucoup plus un capital de savoir-faire que monétaire, et qui ont fait souche au Québec. Leur apport est bien différent de celui des multinationales.

À propos de la Franco-Américanie et de ses relations avec le Q uébec, cet état de la question incite à se dégager du cadre dans lequel on a trop souvent enfermé son étude: celui du discours des élites cléricales et nationalistes, celui de la reproduction des institutions et celui de la N ouvelle-Angleterre. Il invite à tenir compte des rapports économiques, des éléments de formation et d'acquisition de connaissances des transferts culturels et cela, dans le cadre géographique de l'ensemble des 
États-Unis. Par ailleurs, il remet en question l'association étroite qui est souvent faite entre rapatriement et colonisation en montrant que le phénomène du rapatriement a touché un éventail de couches sociales et de milieux d'activités.

Notre bilan a donné une certaine cohérence à des informations souvent éparses, mais il a aussi indiqué les limites de nos connaissances II permet cependant d'identifier des pistes de recherches prometteuses. Notre utilisation, encore limitée, des répertoires biographiques en indique une: la biographie collective des élites américaines et francoaméricaines au Québec. II existe un grand nombre de sources de ce genre, certaines portant sur une région, d'autres sur une profession. Leur dépouillement systématique fournirait la possibilité d'établir une cartographie des membres de ces élites et d'identifier leurs trajectoires, tant spatiales que professionnelles. II pourrait aussi conduire à des études sectorielles plus poussées grâce au jumelage avec d'autres types de sources.

Il faudrait aussi pouvoir multiplier les enquêtes, comme celles de Ramirez et de Rodrigue, portant sur des quartiers urbains ou des paroisses rurales afin d'obtenir une image plus fine et plus détaillée de la présence franco-américaine au Québec et de son impact sur la société ambiante. Les manuscrits des recensements constituent un bon point de départ d'une telle démarche, mais il faudrait aussi mettre à profit les sources foncières et notariales, les M émoires d'anciens et la correspondance privée. 